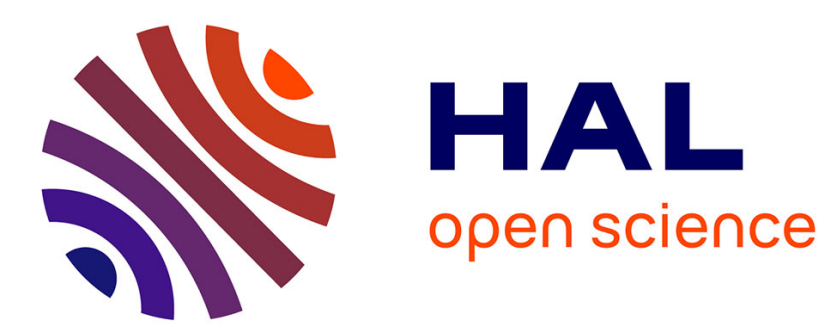

\title{
Explicit examples of nonsolvable weakly hyperbolic operators with real coefficients
}

\author{
Karel Pravda-Starov
}

\section{To cite this version:}

Karel Pravda-Starov. Explicit examples of nonsolvable weakly hyperbolic operators with real coefficients. 2006. hal-00109894

\section{HAL Id: hal-00109894 \\ https://hal.science/hal-00109894}

Preprint submitted on 9 Nov 2006

HAL is a multi-disciplinary open access archive for the deposit and dissemination of scientific research documents, whether they are published or not. The documents may come from teaching and research institutions in France or abroad, or from public or private research centers.
L'archive ouverte pluridisciplinaire HAL, est destinée au dépôt et à la diffusion de documents scientifiques de niveau recherche, publiés ou non, émanant des établissements d'enseignement et de recherche français ou étrangers, des laboratoires publics ou privés. 


\title{
EXPLICIT EXAMPLES OF NONSOLVABLE WEAKLY HYPERBOLIC OPERATORS WITH REAL COEFFICIENTS
}

\author{
KAREL Pravda-Starov
}

University of California, Berkeley

\begin{abstract}
We give in this paper two explicit examples of nonsolvable weakly hyperbolic operators with real coefficients in two-space-dimensions.
\end{abstract}

Keywords. Solvability, doubly characteristic operators, weakly hyperbolic operators.

MSC. 35A07.

\section{Introduction}

We provide here some explicit examples of nonsolvable weakly hyperbolic operators with real coefficients. These are, with $(t, x, y) \in \mathbb{R}^{3}$,

$$
\begin{gathered}
L_{1}=\partial_{t}\left(\partial_{t}+y \partial_{x}\right)+\partial_{y}, \\
L_{2}=\partial_{t}^{2}-H(-y)|y|^{k} \partial_{x}^{2}+\partial_{y}, k \in \mathbb{N}^{*}, H=\mathbb{1}_{\mathbb{R}_{+}},
\end{gathered}
$$

where the notation $\mathbb{1}_{\mathbb{R}_{+}}$stands for the characteristic function of the set $\mathbb{R}_{+}$. Both examples are weakly hyperbolic operators in two-space-dimensions. The operator $L_{1}$ has affine coefficients and the operator $L_{2}$ has coefficients in $C^{k-1}$. Y.V. Egorov gave in [2] an example of a nonsolvable weakly hyperbolic operator in one-space-dimension with a quite complicated expression. Although our examples are 2-space-dimensional, we feel that their simple expression is worth noticing.

Let us begin by recalling some results about solvability for pseudodifferential operators with real principal symbols. Let $L$ be a classical pseudo-differential operator on an open set $\Omega$ of $\mathbb{R}^{n}$ with a real principal symbol $a_{m}$. The double characteristic set is defined as

$$
\Sigma_{2}=\left\{(x, \xi) \in \dot{T}^{*}(\Omega): a_{m}(x, \xi)=0, d_{\xi} a_{m}(x, \xi)=0\right\},
$$

where $\dot{T}^{*}(\Omega)$ is the cotangent bundle minus the zero section.

- If the set $\Sigma_{2}$ is empty, the operator $L$ is of strong-real-principal-type and local solvability with a loss of one derivative holds according to the theorem 26.1.7 in [4]. 
- In the case where the operator $L$ has a real principal symbol $a_{m}$ such that its subprincipal symbol $a_{m-1}^{s}$ satisfies

$$
a_{m}(x, \xi)=0, d_{\xi} a_{m}(x, \xi)=0 \Rightarrow \operatorname{Im} a_{m-1}^{s}(x, \xi) \neq 0,
$$

if $(x, \xi) \in \dot{T}^{*}(\Omega)$, N. Lerner has proved in the theorem 1.1 of [5] that there is also local solvability with a loss of one derivative. For example, this is the case of most of the operators of the type

$$
A B+C,
$$

where $A, B, C$ are smooth real vector fields in $\mathbb{R}^{3}$ such that $A, B$ and $[A, B]$ are linearly independent, for which F. Treves has shown in [8] that they are locally solvable.

- If we now assume that the set

$$
\tilde{\Sigma}_{2}=\left\{(x, \xi) \in \dot{T}^{*}(\Omega): a_{m}(x, \xi)=0, d_{\xi} a_{m}(x, \xi)=0, \operatorname{Im} a_{m-1}^{s}(x, \xi)=0\right\},
$$

is non-empty, different situations can occur. For example, for the class of operators $A B+C$ studied by F. Treves in [8], the set $\tilde{\Sigma}_{2}$ can be non-empty, but the special structure of the principal symbol which appears as a product $p q$ with $\{p, q\} \neq 0$ at $p=q=0$, allows this author to obtain a solvability result with a loss of derivatives. The set $\tilde{\Sigma}_{2}$ can also be non-empty in the cases studied by G.A. Mendoza and G.A. Uhlmann in [7], for which they introduced the additional assumption $\operatorname{Sub}(\mathcal{P})$, also with a product structure (of involutive type) for the principal symbol.

Let us mention that there is a nice example in [1] of an operator verifying (1.0.1), which is therefore locally solvable although a quasi-homogeneous version of condition $(\Psi)$ is violated in that case. For the operators $L_{1}$ and $L_{2}$, the set $\tilde{\Sigma}_{2}$ is non-empty. The nonsolvability in any neighbourhood of 0 in $\mathbb{R}^{3}$ of the operator $L_{1}$ is a consequence of the result of nonsolvability proved by G.A. Mendoza and G.A. Uhlmann in the theorem 1.2 of [7]. We verify in this case that the operator $L_{1}$ violates the condition $\operatorname{Sub}(\mathcal{P})$ defined in [6] and [7]. To prove the nonsolvability in any neighbourhood of 0 for the operator with $C^{k-1}$ coefficients $L_{2}$, we prove by building a quasimode that no a priori estimates of the following type could hold

$$
\begin{aligned}
& \exists C_{0}>0, \exists N_{0} \in \mathbb{N}, \exists V_{0} \text { an open neighbourhood of } 0 \text { in } \mathbb{R}^{3} \text { such that } \\
& \qquad u \in C_{0}^{\infty}\left(V_{0}\right), C_{0}\left\|L_{2}^{*} u\right\|_{(k-3)} \geq\|u\|_{\left(-N_{0}\right)},
\end{aligned}
$$

where the notation $\|\cdot\|_{(s)}$ stands for the $H^{s}\left(\mathbb{R}^{3}\right)$ Sobolev norm. This fact induces that there do not exist an integer $N_{0} \in \mathbb{N}$ and an open neighbourhood $V_{0}$ of 0 in $\mathbb{R}^{3}$ such that for all $f \in H^{N_{0}}\left(V_{0}\right)$, there exists $u \in H^{-k+3}\left(\mathbb{R}^{3}\right)$ such that

$$
L_{2} u=f,
$$


on $V_{0}$ (let us notice that the quantity $L_{2} u$ is well defined for $u \in H^{-k+3}\left(\mathbb{R}^{3}\right)$ ). Indeed if it was the case, we would have using similar arguments to the ones given by L. Hörmander in the proof of Lemma 26.4.5 in [4] that for all $v \in C_{0}^{\infty}\left(V_{0}\right)$,

$$
\left|(f, v)_{L^{2}\left(V_{0}\right)}\right|=\left|\left(L_{2} u, v\right)\right|=\left|\left(u, L_{2}^{*} v\right)\right| \leq\|u\|_{(-k+3)}\left\|L_{2}^{*} v\right\|_{(k-3)} .
$$

Let us consider

$$
\begin{aligned}
T_{v}: H^{N_{0}}\left(V_{0}\right) & \rightarrow \mathbb{C} \\
f & \mapsto(f, v)_{L^{2}\left(V_{0}\right)},
\end{aligned}
$$

for $v$ in $C_{0}^{\infty}\left(V_{0}\right)$. We deduce from the previous estimate that for all $f$ in $H^{N_{0}}\left(V_{0}\right)$, there exists $u \in H^{-k+3}\left(\mathbb{R}^{3}\right)$ such that

$$
\sup _{v \in W}\left|T_{v}(f)\right| \leq\|u\|_{(-k+3)}<+\infty
$$

if $W=\left\{v \in C_{0}^{\infty}\left(V_{0}\right),\left\|L_{2}^{*} v\right\|_{(k-3)} \leq 1\right\}$. Since $T_{v}$ is a bounded linear form for $v$ in $W$, we deduce from the uniform boundedness principle that there exists a positive constant $C_{0}$ such that

$$
\sup _{v \in W}\left\|T_{v}\right\| \leq C_{0}<+\infty .
$$

It follows that for all $f \in H^{N_{0}}\left(V_{0}\right)$ and $v \in C_{0}^{\infty}\left(V_{0}\right),\left\|L_{2}^{*} v\right\|_{(k-3)} \leq 1$, we have

$$
\left|(f, v)_{L^{2}\left(V_{0}\right)}\right| \leq C_{0}\|f\|_{\left(N_{0}\right)},
$$

which induces by homogeneity that for all $f \in H^{N_{0}}\left(V_{0}\right)$ and $v \in C_{0}^{\infty}\left(V_{0}\right)$,

$$
\left|(f, v)_{L^{2}\left(V_{0}\right)}\right| \leq C_{0}\|f\|_{\left(N_{0}\right)}\left\|L_{2}^{*} v\right\|_{(k-3)},
$$

if $\left\|L_{2}^{*} v\right\|_{(k-3)} \neq 0$. According to (1.0.2), we notice that this estimate (1.0.3) is also fulfilled if $\left\|L_{2}^{*} v\right\|_{(k-3)}=0$. Using now that $\left\|T_{v}\right\|=\|v\|_{\left(-N_{0}\right)}$ for all $v$ in $C_{0}^{\infty}\left(V_{0}\right)$, we obtain from (1.0.3) that the following estimate

$$
\forall v \in C_{0}^{\infty}\left(V_{0}\right), C_{0}\left\|L_{2}^{*} v\right\|_{(k-3)} \geq\|v\|_{\left(-N_{0}\right)},
$$

holds, which is not possible according to our result.

\section{Nonsolvability of the operator $L_{1}$}

The operator $L_{1}$ is defined in standard quantization (and also in Weyl quantization) by the symbol

$$
p(t, x, y ; \tau, \xi, \eta)=-\tau(\tau+y \xi)+i \eta .
$$


We first notice that its principal symbol, $p_{2}=-\tau(\tau+y \xi)$, is real and that the doubly characteristic set

$$
\Sigma_{2}\left(L_{1}\right)=\left\{(t, x, y ; \tau, \xi, \eta) \in \dot{T}^{*}\left(\mathbb{R}^{3}\right): p_{2}=0, d_{\tau, \xi, \eta} p_{2}=0\right\},
$$

where $\dot{T}^{*}\left(\mathbb{R}^{3}\right)$ stands for the cotangent bundle minus the zero section, is not empty since

$$
\begin{aligned}
& \Sigma_{2}\left(L_{1}\right)=\{(t, x, y ; \tau, \xi, \eta)\left.\in \dot{T}^{*}\left(\mathbb{R}^{3}\right): y=\tau=0,(\xi, \eta) \neq(0,0)\right\} \\
& \cup\left\{(t, x, y ; \tau, \xi, \eta) \in \dot{T}^{*}\left(\mathbb{R}^{3}\right): \tau=\xi=0, \eta \neq 0\right\} .
\end{aligned}
$$

Let us consider the two real-valued symbols $q=-\tau$ and $s=\tau+y \xi$, we have $p_{2}=q s$. The set $\Sigma_{2}\left(L_{1}\right)$ is a submanifold of codimension 2 near the point $\nu_{0}=\left(t_{0}, x_{0}, 0 ; 0,1,0\right) \in \Sigma_{2}\left(L_{1}\right)$ if $t_{0}, x_{0} \in \mathbb{R}$, which is involutive since

$$
\begin{aligned}
\left(T_{\nu} \Sigma_{2}\left(L_{1}\right)\right)^{\sigma}=\left\{(t, x, y ; \tau, \xi, \eta) \in \mathbb{R}^{6}: x=y=\tau=\xi=0\right\} & \\
\subset T_{\nu} \Sigma_{2}\left(L_{1}\right)=\{(t, x, y ; \tau, \xi, \eta) & \left.\in \mathbb{R}^{6}: y=\tau=0\right\}
\end{aligned}
$$

for all $\nu$ belonging to a neighbourhood of $\nu_{0}$ in $\Sigma_{2}\left(L_{1}\right)$ if $T_{\nu} \Sigma_{2}\left(L_{1}\right)$ stands for the tangent plane of $\Sigma_{2}\left(L_{1}\right)$ in $\nu$. We also notice that the Hamilton vector fields $H_{q}, H_{s}$ and the radial vector field $r$, which are equal to

$$
H_{q}=-\frac{\partial}{\partial t}, H_{s}=\frac{\partial}{\partial t}-\xi \frac{\partial}{\partial \eta}, r=\xi \frac{\partial}{\partial \xi}+\eta \frac{\partial}{\partial \eta},
$$

at points in $\Sigma_{2}\left(L_{1}\right)$ near $\nu_{0}$, are independent and that the imaginary part of the subprincipal symbol, $p_{1}^{s}=i \eta$, changes sign at the first order in 0 along the following bicharacteristic of the symbol $s$,

$$
\left\{\begin{array}{l}
\gamma^{\prime}(t)=H_{s}(\gamma(t)) \\
\gamma(0)=\nu_{0}
\end{array}\right.
$$

since $\operatorname{Im} p_{1}^{s}\left(\nu_{0}\right)=0$ and

$$
\begin{aligned}
& \left.\frac{d}{d t}\left[\operatorname{Im} p_{1}^{s}(\gamma(t))\right]\right|_{t=0}=\left.d \operatorname{Im} p_{1}^{s}(\gamma(t)) \cdot H_{s}(\gamma(t))\right|_{t=0}= \\
& \left.\sigma\left(H_{s}(\gamma(t)), H_{\operatorname{Im} p_{1}^{s}}(\gamma(t))\right)\right|_{t=0}=\left.\left\{s, \operatorname{Im} p_{1}^{s}\right\}(\gamma(t))\right|_{t=0}=-1 \neq 0 .
\end{aligned}
$$

It follows that the condition $\operatorname{Sub}(\mathcal{P})$ defined by G.A. Mendoza and G.A. Uhlmann in [7] is violated and we deduce from Theorem 1.2 in [7] that the operator $L_{1}$ is not locally solvable at $\nu_{0} \in \Sigma_{2}\left(L_{1}\right)$, which induces that the operator $L_{1}$ is nonsolvable in any neighbourhood of 0 in $\mathbb{R}^{3}$. 


\section{Nonsolvability of the operator $L_{2}$}

The second operator $L_{2}$ that we study, is defined in standard quantization (and also in Weyl quantization) by the symbol

$$
p=i \eta+\left(\theta_{k}(y) \xi^{2}-\tau^{2}\right)=i\left(\eta+i\left(\tau^{2}-\theta_{k}(y) \xi^{2}\right)\right),
$$

where $\theta_{k}$ is the $C^{k-1}(\mathbb{R}, \mathbb{R})$ function defined for $k \in \mathbb{N}^{*}$ by

$$
\theta_{k}(y)=(-1)^{k} y^{k} H(-y) \text { if } H=\mathbb{1}_{\mathbb{R}_{+}},
$$

where the notation $\mathbb{1}_{X}$ stands for characteristic function of the set $X$. We notice that its principal symbol, $p_{2}=\theta_{k}(y) \xi^{2}-\tau^{2}$, is a real $C^{k-1}$ symbol and that the doubly characteristic set

$$
\begin{aligned}
\Sigma_{2}\left(L_{2}\right)= & \left\{(t, x, y ; \tau, \xi, \eta) \in \dot{T}^{*}\left(\mathbb{R}^{3}\right): p_{2}=0, d_{\tau, \xi, \eta} p_{2}=0\right\} \\
= & \left\{(t, x, y ; \tau, \xi, \eta) \in \dot{T}^{*}\left(\mathbb{R}^{3}\right): \tau=0, y \in \mathbb{R}_{+}\right\} \\
& \cup\left\{(t, x, y ; \tau, \xi, \eta) \in \dot{T}^{*}\left(\mathbb{R}^{3}\right): \tau=\xi=0\right\},
\end{aligned}
$$

is not empty. This set contains some points, $(t, x, 0 ; 0, \pm 1,0) \in \Sigma_{2}\left(L_{2}\right)$, where the imaginary part of the subprincipal symbol vanishes, $p_{1}^{s}=i \eta$. Then, we notice that since the function $y \mapsto \tau^{2}-\theta_{k}(y) \xi^{2}$ changes sign from - to + whenever $\tau \xi \neq 0$ if $y$ increases, the symbol $p$ violates a quasihomogeneous version of the condition $(\Psi)$.

\subsection{Construction of a quasimode}

Let us consider $N_{0} \in \mathbb{N}$,

$$
\begin{gathered}
\psi_{1} \in C_{0}^{\infty}\left(\mathbb{R}^{2}, \mathbb{R}\right), \operatorname{supp} \psi_{1} \subset[1,4]^{2}, \psi_{1}=1 \text { on }[2,3]^{2}, \\
\chi_{0} \in C_{0}^{\infty}(\mathbb{R}, \mathbb{R}), \operatorname{supp} \chi_{0} \subset[-1,1], \chi_{0}=1 \text { on }[-1 / 2,1 / 2],
\end{gathered}
$$

some positive parameters $\alpha$ and $\mu$ such that

$$
\frac{1}{k}<\alpha<\frac{2}{k} \text { and } \frac{2}{k}<\mu<\alpha+\frac{1}{k},
$$

where $k$ is the integer appearing in the definition of the operator $L_{2}$. We set for all $\lambda \geq 1$,

$$
\psi_{\lambda}(\tau, \xi)=\lambda^{-\frac{1}{2}-\alpha} \psi_{1}\left(\lambda^{-\alpha} \tau, \lambda^{-1-\alpha} \xi\right) .
$$

Let us note supp $\chi_{0}\left(\lambda^{\mu}\left(\cdot+\left(\tau \xi^{-1} \lambda^{-1}\right)^{\frac{2}{k}}\right)\right)$ for the support of the function

$$
y \mapsto \chi_{0}\left(\lambda^{\mu}\left(y+\left(\tau \xi^{-1} \lambda^{-1}\right)^{\frac{2}{k}}\right)\right) .
$$

Since using (3.1.2), we have for all $(\tau, \xi) \in[1,4]^{2}$ and $\lambda \geq 1$,

$$
\begin{array}{r}
\operatorname{supp} \chi_{0}\left(\lambda^{\mu}\left(\cdot+\left(\tau \xi^{-1} \lambda^{-1}\right)^{\frac{2}{k}}\right)\right) \subset\left\{y \in \mathbb{R}:\left|y+\left(\tau \xi^{-1} \lambda^{-1}\right)^{\frac{2}{k}}\right| \leq \lambda^{-\mu}\right\} \\
\subset\left\{y \in \mathbb{R}:-\lambda^{-\mu}-4^{\frac{2}{k}} \lambda^{-\frac{2}{k}} \leq y \leq \lambda^{-\mu}-4^{-\frac{2}{k}} \lambda^{-\frac{2}{k}}\right\},
\end{array}
$$


it follows from (3.1.3), $2 / k<\mu$, that we can find a constant $\lambda_{0} \geq 1$ and some positive constants $c_{1}, c_{2}$ such that $c_{1}>c_{2}$ and for all $(\tau, \xi) \in[1,4]^{2}$, $\lambda \geq \lambda_{0}$

$$
\operatorname{supp} \chi_{0}\left(\lambda^{\mu}\left(\cdot+\left(\tau \xi^{-1} \lambda^{-1}\right)^{\frac{2}{k}}\right)\right) \subset\left\{y \in \mathbb{R}:-c_{1} \lambda^{-\frac{2}{k}} \leq y \leq-c_{2} \lambda^{-\frac{2}{k}}\right\} .
$$

Let us notice that since

$$
L_{2}^{*}=-\partial_{y}+\theta_{k}(y) D_{x}^{2}-D_{t}^{2}, \theta_{k}(y)=(-1)^{k} y^{k} H(-y), H=\mathbb{1}_{\mathbb{R}_{+}},
$$

with $D_{x}=i^{-1} \partial_{x}, D_{t}=i^{-1} \partial_{t}$, and since the function $y \mapsto \tau^{2}-\theta_{k}(y) \xi^{2}$ changes sign from - to + at $y=-\left(\tau \xi^{-1}\right)^{2 / k}$ if $(\tau, \xi) \in \mathbb{R}_{+}^{*} \times \mathbb{R}_{+}^{*}$, we can find a non-negative phase function $\Phi_{1}$, which satisfies the equation

$$
\begin{aligned}
& \left(-\partial_{y}+\theta_{k}(y) \xi^{2}-\tau^{2}\right)\left(e^{-\Phi_{1}(\tau, \xi, y)}\right) \\
= & \left(\partial_{y} \Phi_{1}(\tau, \xi, y)+\theta_{k}(y) \xi^{2}-\tau^{2}\right) e^{-\Phi_{1}(\tau, \xi, y)}=0,
\end{aligned}
$$

defined for all $y \in \mathbb{R}_{-}^{*}$ and $(\tau, \xi) \in \mathbb{R}_{+}^{*} \times \mathbb{R}_{+}^{*}$ by

$$
\Phi_{1}(\tau, \xi, y)=\int_{-\left(\tau \xi^{-1}\right)^{\frac{2}{k}}}^{y}\left(\tau^{2}-\theta_{k}(s) \xi^{2}\right) d s .
$$

Indeed, since from (3.1.6) and (3.1.8),

$$
\frac{\partial^{2} \Phi_{1}}{\partial y^{2}}(\tau, \xi, y)=k(-y)^{k-1} \xi^{2} \geq 0
$$

if $y \in \mathbb{R}_{-}^{*}$ and $(\tau, \xi) \in \mathbb{R}_{+}^{*} \times \mathbb{R}_{+}^{*}$, the function $y \mapsto \Phi_{1}(\tau, \xi, y)$ is convex on $\mathbb{R}_{-}^{*}$ and we deduce from the fact

$$
\Phi_{1}\left(\tau, \xi,-\left(\tau \xi^{-1}\right)^{\frac{2}{k}}\right)=0, \frac{\partial \Phi_{1}}{\partial y}\left(\tau, \xi,-\left(\tau \xi^{-1}\right)^{\frac{2}{k}}\right)=0
$$

and from the Taylor formula that

$$
\Phi_{1}(\tau, \xi, y)=\left(y+\left(\tau \xi^{-1}\right)^{\frac{2}{k}}\right)^{2} k \xi^{2} \int_{0}^{1}(1-\theta)\left(\left(\tau \xi^{-1}\right)^{\frac{2}{k}}(1-\theta)-\theta y\right)^{k-1} d \theta
$$

if $y \in \mathbb{R}_{-}^{*}$ and $(\tau, \xi) \in \mathbb{R}_{+}^{*} \times \mathbb{R}_{+}^{*}$. The property of non-negativity of the function $\Phi_{1}$ on $\mathbb{R}_{+}^{*} \times \mathbb{R}_{+}^{*} \times \mathbb{R}_{-}^{*}$ is clear on the formula (3.1.10). We also set for all $y \in \mathbb{R}_{-}^{*},(\tau, \xi) \in \mathbb{R}_{+}^{*} \times \mathbb{R}_{+}^{*}$ and $\lambda \geq 1$,

$$
\begin{aligned}
\Phi_{\lambda}(\tau, \xi, y) & =\Phi_{1}\left(\lambda^{\alpha} \tau, \lambda^{1+\alpha} \xi, y\right) \\
& =\int_{-\left(\tau \xi^{-1} \lambda^{-1}\right)^{\frac{2}{k}}}^{y}\left(\lambda^{2 \alpha} \tau^{2}-\theta_{k}(s) \lambda^{2+2 \alpha} \xi^{2}\right) d s,
\end{aligned}
$$


which is also a non-negative function. A direct computation shows from (3.1.6) and (3.1.11) that for all $y \in \mathbb{R}_{-}^{*},(\tau, \xi) \in \mathbb{R}_{+}^{*} \times \mathbb{R}_{+}^{*}$ and $\lambda \geq 1$,

$$
\Phi_{\lambda}(\tau, \xi, y)=\lambda^{2 \alpha} \tau^{2} y+\frac{k}{k+1} \lambda^{2 \alpha-\frac{2}{k}} \tau^{2+\frac{2}{k}} \xi^{-\frac{2}{k}}+\frac{(-1)^{k+1}}{k+1} \lambda^{2+2 \alpha} \xi^{2} y^{k+1} .
$$

We can now define for all $\lambda \geq \lambda_{0}$ the function $u_{\lambda}$ defined by

$$
u_{\lambda}(t, x, y)=\frac{1}{(2 \pi)^{2}} \int_{\mathbb{R}^{2}} e^{i(x \xi+t \tau)} \psi_{\lambda}(\tau, \xi) \chi_{0}\left(\lambda^{\mu}\left(y+\left(\tau \xi^{-1}\right)^{\frac{2}{k}}\right)\right) e^{-\Phi_{1}(\tau, \xi, y)} d \tau d \xi .
$$

If we note $\mathcal{F}_{t, x}$ the Fourier transform in the variables $t, x$, it follows from (3.1.13) that

$$
\begin{aligned}
U_{\lambda}(\tau, \xi, y) & =\left(\mathcal{F}_{t, x} u_{\lambda}\right)(\tau, \xi, y) \\
& =\psi_{\lambda}(\tau, \xi) \chi_{0}\left(\lambda^{\mu}\left(y+\left(\tau \xi^{-1}\right)^{\frac{2}{k}}\right)\right) e^{-\Phi_{1}(\tau, \xi, y)}
\end{aligned}
$$

and we can notice from (3.1.1), (3.1.4), (3.1.5) and the change of variables $(\tilde{\tau}, \tilde{\xi})=\left(\lambda^{-\alpha} \tau, \lambda^{-1-\alpha} \xi\right)$ that for all $(\tau, \xi) \in \operatorname{supp} \psi_{\lambda}$ and $\lambda \geq \lambda_{0}$,

$$
\operatorname{supp} U_{\lambda}(\tau, \xi, \cdot) \subset \mathbb{R}_{-}^{*} \text {. }
$$

In view of (3.1.10), (3.1.14) and (3.1.15), it follows that the family $\left(u_{\lambda}\right)_{\lambda \geq \lambda_{0}}$ belongs to the space $C^{\infty}\left(\mathbb{R}_{y}, \mathcal{S}\left(\mathbb{R}_{t, x}^{2}\right)\right.$ ) (because (3.1.1) and (3.1.4) imply that the function $\psi_{\lambda}$ has a compact support in $\left.\mathbb{R}_{+}^{*} \times \mathbb{R}_{+}^{*}\right)$ and has its support included in the set $\mathbb{R}_{t, x}^{2} \times\left(\mathbb{R}_{y}\right)_{-}$. We deduce from this fact and (3.1.6) that

$$
L_{2}^{*} u_{\lambda} \in C^{\infty}\left(\mathbb{R}_{t, x, y}^{3}\right)
$$

and a direct computation using (3.1.6), (3.1.7) and (3.1.14) gives that

$$
\begin{aligned}
\mathcal{F}_{t, x}\left(L_{2}^{*} u_{\lambda}\right) & =-\partial_{y} U_{\lambda}(\tau, \xi, y)+\left(\theta_{k}(y) \xi^{2}-\tau^{2}\right) U_{\lambda}(\tau, \xi, y) \\
& =-\lambda^{\mu} \psi_{\lambda}(\tau, \xi) \chi_{0}^{\prime}\left(\lambda^{\mu}\left(y+\left(\tau \xi^{-1}\right)^{\frac{2}{k}}\right)\right) e^{-\Phi_{1}(\tau, \xi, y)} .
\end{aligned}
$$

\subsection{Upper bound for $\left\|L_{2}^{*} u_{\lambda}\right\|_{\left(N_{0}\right)}$}

From (3.1.17) and Parseval's formula, we notice that to obtain an upper bound for the quantity $\left\|L_{2}^{*} u_{\lambda}\right\|_{\left(N_{0}\right)}$, it is enough to get an upper bound for the quantities

$$
\begin{aligned}
& A_{j_{1}, j_{2}, j_{3}, j_{4}}(\lambda)= \\
& \left\|\lambda^{\mu\left(j_{1}+1\right)} \chi_{0}^{\left(j_{1}+1\right)}\left(\lambda^{\mu}\left(y+\left(\tau \xi^{-1}\right)^{\frac{2}{k}}\right)\right) \tau^{j_{2}} \xi^{j_{3}} \psi_{\lambda}(\tau, \xi) \partial_{y}^{j_{4}}\left(e^{-\Phi_{1}(\tau, \xi, y)}\right)\right\|_{L^{2}\left(\mathbb{R}^{3}\right)},
\end{aligned}
$$


where $\left(j_{1}, j_{2}, j_{3}, j_{4}\right) \in \mathbb{N}^{4}$ are some integers such that $j_{1}+j_{2}+j_{3}+j_{4}=N_{0}$. Using a change of variables, (3.1.4) and (3.1.11), we obtain that

$$
\begin{aligned}
A_{j_{1}, j_{2}, j_{3}, j_{4}}(\lambda)^{2}= & \lambda^{2 \mu\left(j_{1}+1\right)+2 \alpha j_{2}+2 j_{3}(1+\alpha)} \int_{\mathbb{R}^{3}}\left[\chi_{0}^{\left(j_{1}+1\right)}\left(\lambda^{\mu}\left(y+\left(\tau \xi^{-1} \lambda^{-1}\right)^{\frac{2}{k}}\right)\right)^{2}\right. \\
& \left.\times \tau^{2 j_{2}} \xi^{2 j_{3}} \psi_{1}(\tau, \xi)^{2}\left|\partial_{y}^{j_{4}}\left(e^{-\Phi_{\lambda}(\tau, \xi, y)}\right)\right|^{2}\right] d y d \tau d \xi . \quad(3.2 .2)
\end{aligned}
$$

Let us stress the fact that from (3.1.1), (3.1.5), (3.1.10) and (3.1.11), if $(\tau, \xi) \in \operatorname{supp} \psi_{1}$ and $\lambda \geq \lambda_{0}$, the function $\Phi_{\lambda}(\tau, \xi, \cdot)$ is $C^{\infty}$ on

$$
\operatorname{supp} \chi_{0}^{\left(j_{1}+1\right)}\left(\lambda^{\mu}\left(\cdot+\left(\tau \xi^{-1} \lambda^{-1}\right)^{\frac{2}{k}}\right)\right) \subset \mathbb{R}_{-}^{*} .
$$

Thus, the expression (3.2.2) is well-defined. We need now the following lemma.

Lemma 3.2.1 For all $\nu \in \mathbb{N}^{3}$, there exist some functions $a_{l}, l=0, \ldots,|\nu|(k+$ $1)$, which are polynomial in $\mathbb{R}^{4}$ and some constants $\beta_{l}, l=0, \ldots,|\nu|(k+1)$ verifying

$$
\beta_{l} \leq 2|\nu|(\alpha+1),
$$

such that for all $y \in \mathbb{R}_{-}^{*},(\tau, \xi) \in \mathbb{R}_{+}^{*} \times \mathbb{R}_{+}^{*}$ and $\lambda \geq 1$,

$$
\partial_{\tau, \xi, y}^{\nu}\left(e^{-\Phi_{\lambda}}\right)=e^{-\Phi_{\lambda}} \sum_{l=0}^{|\nu|(k+1)} a_{l}\left(\tau^{\frac{1}{k}}, \tau^{-\frac{1}{k}}, \xi^{\frac{1}{k}}, \xi^{-\frac{1}{k}}\right) y^{l} \lambda^{\beta_{l}} .
$$

Proof. We prove this lemma by induction on $|\nu|$. If $|\nu|=0$, the expression (3.2.3) holds with $a_{0}=1$ and $\beta_{0}=0$. Let us assume now that for $\nu \in \mathbb{N}^{3}$, there exist some functions $a_{l}, l=0, \ldots,|\nu|(k+1)$, which are polynomial in $\mathbb{R}^{4}$ and some constants $\beta_{l}, l=0, \ldots,|\nu|(k+1)$ verifying $\beta_{l} \leq 2|\nu|(\alpha+1)$ such that for all $y \in \mathbb{R}_{-}^{*},(\tau, \xi) \in \mathbb{R}_{+}^{*} \times \mathbb{R}_{+}^{*}, \lambda \geq 1$, the expression (3.2.3) holds. Since from (3.1.12), we have for all $y \in \mathbb{R}_{-}^{*},(\tau, \xi) \in \mathbb{R}_{+}^{*} \times \mathbb{R}_{+}^{*}$ and $\lambda \geq 1$,

$$
\begin{gathered}
\partial_{y} \Phi_{\lambda}(\tau, \xi, y)=\lambda^{2 \alpha} \tau^{2}+(-1)^{k+1} \lambda^{2+2 \alpha} \xi^{2} y^{k} \\
\partial_{\tau} \Phi_{\lambda}(\tau, \xi, y)=2 \lambda^{2 \alpha} \tau y+2 \lambda^{2 \alpha-\frac{2}{k}} \xi^{-\frac{2}{k}} \tau^{1+\frac{2}{k}} \\
\partial_{\xi} \Phi_{\lambda}(\tau, \xi, y)=\frac{2}{k+1}\left[-\lambda^{2 \alpha-\frac{2}{k}} \tau^{2+\frac{2}{k}} \xi^{-\frac{2}{k}-1}+(-1)^{k+1} \lambda^{2+2 \alpha} \xi y^{k+1}\right] .
\end{gathered}
$$

We have also for all $y \in \mathbb{R}_{-}^{*},(\tau, \xi) \in \mathbb{R}_{+}^{*} \times \mathbb{R}_{+}^{*}$ and $\lambda \geq 1$,

$$
\begin{aligned}
\partial_{y}\left(e^{-\Phi_{\lambda}} \sum_{l=0}^{|\nu|(k+1)} a_{l}\left(\tau^{\frac{1}{k}}, \tau^{-\frac{1}{k}}, \xi^{\frac{1}{k}}, \xi^{-\frac{1}{k}}\right) y^{l} \lambda^{\beta_{l}}\right)= \\
e^{-\Phi_{\lambda}} \sum_{l=0}^{|\nu|(k+1)} a_{l}\left(\tau^{\frac{1}{k}}, \tau^{-\frac{1}{k}}, \xi^{\frac{1}{k}}, \xi^{-\frac{1}{k}}\right)\left[-\left(\partial_{y} \Phi_{\lambda}\right) y^{l}+l y^{l-1}\right] \lambda^{\beta_{l}},
\end{aligned}
$$




$$
\begin{aligned}
& \partial_{\tau}\left(e^{-\Phi_{\lambda}} \sum_{l=0}^{|\nu|(k+1)} a_{l}\left(\tau^{\frac{1}{k}}, \tau^{-\frac{1}{k}}, \xi^{\frac{1}{k}}, \xi^{-\frac{1}{k}}\right) y^{l} \lambda^{\beta_{l}}\right)= \\
& e^{-\Phi_{\lambda}} \sum_{l=0}^{|\nu|(k+1)} \lambda^{\beta_{l}} y^{l}\left[-\left(\partial_{\tau} \Phi_{\lambda}\right) a_{l}\left(\tau^{\frac{1}{k}}, \tau^{-\frac{1}{k}}, \xi^{\frac{1}{k}}, \xi^{-\frac{1}{k}}\right)+\partial_{\tau}\left(a_{l}\left(\tau^{\frac{1}{k}}, \tau^{-\frac{1}{k}}, \xi^{\frac{1}{k}}, \xi^{-\frac{1}{k}}\right)\right)\right]
\end{aligned}
$$

and

$$
\begin{aligned}
& \partial_{\xi}\left(e^{-\Phi_{\lambda}} \sum_{l=0}^{|\nu|(k+1)} a_{l}\left(\tau^{\frac{1}{k}}, \tau^{-\frac{1}{k}}, \xi^{\frac{1}{k}}, \xi^{-\frac{1}{k}}\right) y^{l} \lambda^{\beta_{l}}\right)= \\
& e^{-\Phi_{\lambda}} \sum_{l=0}^{|\nu|(k+1)} \lambda^{\beta_{l}} y^{l}\left[-\left(\partial_{\xi} \Phi_{\lambda}\right) a_{l}\left(\tau^{\frac{1}{k}}, \tau^{-\frac{1}{k}}, \xi^{\frac{1}{k}}, \xi^{-\frac{1}{k}}\right)+\partial_{\xi}\left(a_{l}\left(\tau^{\frac{1}{k}}, \tau^{-\frac{1}{k}}, \xi^{\frac{1}{k}}, \xi^{-\frac{1}{k}}\right)\right)\right] .
\end{aligned}
$$

We deduce from (3.2.4), (3.2.5), (3.2.6), (3.2.7), (3.2.8) and (3.2.9) that if

$$
\tilde{\nu} \in\{\nu+(1,0,0), \nu+(0,1,0), \nu+(0,0,1)\},
$$

there exist some functions $\tilde{a}_{l}, l=0, \ldots,|\tilde{\nu}|(k+1)$, which are polynomial in $\mathbb{R}^{4}$ and some constants $\tilde{\beta}_{l}, l=0, \ldots,|\tilde{\nu}|(k+1)$ verifying $\tilde{\beta}_{l} \leq 2|\tilde{\nu}|(\alpha+1)$ such that for all $y \in \mathbb{R}_{-}^{*},(\tau, \xi) \in \mathbb{R}_{+}^{*} \times \mathbb{R}_{+}^{*}$ and $\lambda \geq 1$,

$$
\partial_{\tau, \xi, y}^{\tilde{\nu}}\left(e^{-\Phi_{\lambda}}\right)=e^{-\Phi_{\lambda}} \sum_{l=0}^{|\tilde{\nu}|(k+1)} \tilde{a}_{l}\left(\tau^{\frac{1}{k}}, \tau^{-\frac{1}{k}}, \xi^{\frac{1}{k}}, \xi^{-\frac{1}{k}}\right) y^{l} \lambda^{\tilde{\beta}_{l}} .
$$

Indeed, let us consider for example the case where $\tilde{\nu}=\nu+(0,0,1)$. We obtain from (3.2.3), (3.2.4) and (3.2.7) the expression

$$
\begin{gathered}
\partial_{\tau, \xi, y}^{\tilde{\nu}}\left(e^{-\Phi_{\lambda}}\right)=e^{-\Phi_{\lambda}} \sum_{l=0}^{|\tilde{\nu}|(k+1)-(k+2)} a_{l+1}\left(\tau^{\frac{1}{k}}, \tau^{-\frac{1}{k}}, \xi^{\frac{1}{k}}, \xi^{-\frac{1}{k}}\right)(l+1) y^{l} \lambda^{\beta_{l+1}} \\
-e^{-\Phi_{\lambda}} \sum_{l=0}^{|\tilde{\nu}|(k+1)-(k+1)} a_{l}\left(\tau^{\frac{1}{k}}, \tau^{-\frac{1}{k}}, \xi^{\frac{1}{k}}, \xi^{-\frac{1}{k}}\right)\left(\tau^{\frac{1}{k}}\right)^{2 k} y^{l} \lambda^{\beta_{l}+2 \alpha} \\
-e^{-\Phi_{\lambda}} \sum_{l=k}^{|\tilde{\nu}|(k+1)-1} a_{l-k}\left(\tau^{\frac{1}{k}}, \tau^{-\frac{1}{k}}, \xi^{\frac{1}{k}}, \xi^{-\frac{1}{k}}\right)(-1)^{k+1}\left(\xi^{\frac{1}{k}}\right)^{2 k} y^{l} \lambda^{\beta_{l-k}+2+2 \alpha},
\end{gathered}
$$

which can be written in the form (3.2.10). Since the power of $\lambda$ is less or equal than $2+2 \alpha$ in every term of the right-hand-side of (3.2.5) and (3.2.6), and that these terms are polynomial functions in the variables $\tau^{\frac{1}{k}}, \tau^{-\frac{1}{k}}, \xi^{\frac{1}{k}}$, 
$\xi^{-\frac{1}{k}}$ and $y$ with a degree in $y$ lower than $k+1$, we have only to use (3.2.5), (3.2.6), (3.2.8), (3.2.9) and the fact that the quantities

$$
\begin{aligned}
\partial_{\tau}\left(a_{l}\left(\tau^{\frac{1}{k}}, \tau^{-\frac{1}{k}}, \xi^{\frac{1}{k}}, \xi^{-\frac{1}{k}}\right)\right)=\frac{1}{k} \tau^{\frac{1}{k}}\left(\tau^{-\frac{1}{k}}\right)^{k}\left(\partial_{1} a_{l}\right)\left(\tau^{\frac{1}{k}}, \tau^{-\frac{1}{k}}, \xi^{\frac{1}{k}}, \xi^{-\frac{1}{k}}\right) \\
\\
\quad-\frac{1}{k}\left(\tau^{-\frac{1}{k}}\right)^{k+1}\left(\partial_{2} a_{l}\right)\left(\tau^{\frac{1}{k}}, \tau^{-\frac{1}{k}}, \xi^{\frac{1}{k}}, \xi^{-\frac{1}{k}}\right)
\end{aligned}
$$

and

$$
\begin{aligned}
\partial_{\xi}\left(a_{l}\left(\tau^{\frac{1}{k}}, \tau^{-\frac{1}{k}}, \xi^{\frac{1}{k}}, \xi^{-\frac{1}{k}}\right)\right)=\frac{1}{k} \xi^{\frac{1}{k}}\left(\xi^{-\frac{1}{k}}\right)^{k}\left(\partial_{3} a_{l}\right)\left(\tau^{\frac{1}{k}}, \tau^{-\frac{1}{k}}, \xi^{\frac{1}{k}}, \xi^{-\frac{1}{k}}\right) \\
-\frac{1}{k}\left(\xi^{-\frac{1}{k}}\right)^{k+1}\left(\partial_{4} a_{l}\right)\left(\tau^{\frac{1}{k}}, \tau^{-\frac{1}{k}}, \xi^{\frac{1}{k}}, \xi^{-\frac{1}{k}}\right),
\end{aligned}
$$

are some polynomial functions in the variables $\tau^{\frac{1}{k}}, \tau^{-\frac{1}{k}}, \xi^{\frac{1}{k}}$ and $\xi^{-\frac{1}{k}}$, to obtain (3.2.10) when $\tilde{\nu}=\nu+(1,0,0)$ or $\tilde{\nu}=\nu+(0,1,0)$. This proves the induction property at the rank $|\nu|+1$ and ends the proof of the lemma 3.2.1.

We deduce from (3.1.5) and the lemma 3.2.1 that there exists a positive constant $C_{j_{4}}$ such that for all $(\tau, \xi) \in[1,4]^{2}, \lambda \geq \lambda_{0}$ and

$$
\begin{gathered}
y \in \operatorname{supp} \chi_{0}\left(\lambda^{\mu}\left(\cdot+\left(\lambda^{-1} \xi^{-1} \tau\right)^{\frac{2}{k}}\right)\right), \\
\left|\partial_{y}^{j_{4}}\left(e^{-\Phi_{\lambda}(\tau, \xi, y)}\right)\right| \leq C_{j_{4}} \lambda^{2 j_{4}(1+\alpha)} e^{-\Phi_{\lambda}(\tau, \xi, y)} .
\end{gathered}
$$

Moreover, we obtain from (3.1.2) and (3.1.5) that for all $(\tau, \xi) \in[1,4]^{2}$ and $\lambda \geq \lambda_{0}$,

$$
\operatorname{supp} \chi_{0}^{\left(j_{1}+1\right)}\left(\lambda^{\mu}\left(\cdot+\left(\lambda^{-1} \xi^{-1} \tau\right)^{\frac{2}{k}}\right)\right) \subset \Omega_{\lambda, \tau, \xi},
$$

if we note

$$
\Omega_{\lambda, \tau, \xi}=\left\{y \in \mathbb{R}_{-}^{*}: 2^{-1} \lambda^{-\mu} \leq\left|y+\left(\lambda^{-1} \xi^{-1} \tau\right)^{\frac{2}{k}}\right| \leq \lambda^{-\mu}\right\} .
$$

Then, we deduce from (3.1.1), (3.2.2), (3.2.11), (3.2.12) and (3.2.13) that for all $\lambda \geq \lambda_{0}$,

$$
\begin{aligned}
A_{j_{1}, j_{2}, j_{3}, j_{4}}(\lambda)^{2} \leq & C_{j_{4}}^{2} \lambda^{2 \mu\left(j_{1}+1\right)+2 j_{2} \alpha+2 j_{3}(1+\alpha)+4 j_{4}(1+\alpha)}\left\|\chi_{0}^{\left(j_{1}+1\right)}\right\|_{L^{\infty}(\mathbb{R})}^{2} \\
& \times \int_{\mathbb{R}^{2}} \tau^{2 j_{2}} \xi^{2 j_{3}} \psi_{1}(\tau, \xi)^{2}\left(\int_{\Omega_{\lambda, \tau, \xi}} e^{-2 \Phi_{\lambda}(\tau, \xi, y)} d y\right) d \tau d \xi \\
\leq & C_{j_{4}}^{2} \lambda^{2 \mu\left(j_{1}+1\right)+2 j_{2} \alpha+2 j_{3}(1+\alpha)+4 j_{4}(1+\alpha)-\mu}\left\|\chi_{0}^{\left(j_{1}+1\right)}\right\|_{L^{\infty}(\mathbb{R})}^{2} \\
& \times \int_{\mathbb{R}^{2}} \tau^{2 j_{2}} \xi^{2 j_{3}} \psi_{1}(\tau, \xi)^{2}\left(\sup _{y \in \Omega_{\lambda, \tau, \xi}} e^{-2 \Phi_{\lambda}(\tau, \xi, y)}\right) d \tau d \xi
\end{aligned}
$$


We obtain from (3.1.10) that for all $y \in \mathbb{R}_{-}^{*}$ and $(\tau, \xi) \in \mathbb{R}_{+}^{*} \times \mathbb{R}_{+}^{*}$,

$$
\begin{array}{r}
\Phi_{1}(\tau, \xi, y)=\left(y+\left(\tau \xi^{-1}\right)^{\frac{2}{k}}\right)^{2} k \xi^{2} \int_{0}^{1}(1-\theta)\left(\left(\tau \xi^{-1}\right)^{\frac{2}{k}}(1-\theta)-\theta y\right)^{k-1} d \theta \\
\geq\left(y+\left(\tau \xi^{-1}\right)^{\frac{2}{k}}\right)^{2} k \xi^{2} \int_{0}^{1}(1-\theta)^{k}\left(\tau \xi^{-1}\right)^{2-\frac{2}{k}} d \theta
\end{array}
$$

which induces that

$$
\Phi_{1}(\tau, \xi, y) \geq \frac{k}{k+1} \xi^{2}\left(\tau \xi^{-1}\right)^{2-\frac{2}{k}}\left(y+\left(\tau \xi^{-1}\right)^{\frac{2}{k}}\right)^{2} .
$$

It follows from (3.1.11) and (3.2.15) that for all $y \in \mathbb{R}_{-}^{*},(\tau, \xi) \in[1,4]^{2}$ and $\lambda \geq 1$,

$$
\begin{aligned}
\Phi_{\lambda}(\tau, \xi, y) & \geq \frac{k}{k+1} \xi^{2}\left(\tau \xi^{-1}\right)^{2-\frac{2}{k}} \lambda^{2 \alpha+\frac{2}{k}}\left(y+\left(\tau \lambda^{-1} \xi^{-1}\right)^{\frac{2}{k}}\right)^{2} \\
& \geq c_{3} \lambda^{2 \alpha+\frac{2}{k}}\left(y+\left(\tau \lambda^{-1} \xi^{-1}\right)^{\frac{2}{k}}\right)^{2}
\end{aligned}
$$

with $c_{3}=4^{\frac{2}{k}-2} k /(k+1)>0$. Thus, we obtain using (3.2.13) and (3.2.16) that for all $(\tau, \xi) \in[1,4]^{2}$ and $\lambda \geq \lambda_{0}$,

$$
\sup _{y \in \Omega_{\lambda, \tau, \xi}} e^{-2 \Phi_{\lambda}(\tau, \xi, y)} \leq e^{-\frac{c_{3}}{2} \lambda^{2\left(\alpha+\frac{1}{k}-\mu\right)}} .
$$

Getting back to (3.2.14), the next proposition follows from (3.1.1), (3.2.1), (3.2.14), (3.2.17) and the fact that from (3.1.3),

$$
\mu<\alpha+\frac{1}{k} \text {. }
$$

Proposition 3.2.1 We have

$$
\left\|L_{2}^{*} u_{\lambda}\right\|_{\left(N_{0}\right)}=O\left(e^{-\frac{c_{3}}{8} \lambda^{2\left(\alpha+\frac{1}{k}-\mu\right)}}\right) \text { when } \lambda \rightarrow+\infty .
$$

\subsection{Lower bound for the quantity $\left\|u_{\lambda}\right\|_{\left(-N_{0}\right)}$}

It follows from (3.1.4), (3.1.11), (3.1.14) and a change of variables that

$$
\begin{aligned}
\left\|u_{\lambda}\right\|_{\left(-N_{0}\right)}^{2}= & \int_{\mathbb{R}^{3}}\left|\psi_{\lambda}(\tau, \xi)\right|^{2}\left|\int_{\mathbb{R}} e^{-i y \eta} \chi_{0}\left(\lambda^{\mu}\left(y+\left(\xi^{-1} \tau\right)^{\frac{2}{k}}\right)\right) e^{-\Phi_{1}(\tau, \xi, y)} d y\right|^{2} \\
& \times\left(1+\eta^{2}+\xi^{2}+\tau^{2}\right)^{-N_{0}} \frac{d \eta d \tau d \xi}{(2 \pi)^{3}} \\
= & \int_{\mathbb{R}^{3}}\left|\psi_{1}(\tau, \xi)\right|^{2}\left|\int_{\mathbb{R}} e^{-i y \eta} \chi_{0}\left(\lambda^{\mu}\left(y+\left(\lambda^{-1} \xi^{-1} \tau\right)^{\frac{2}{k}}\right)\right) e^{-\Phi_{\lambda}(\tau, \xi, y)} d y\right|^{2} \\
& \times\left(1+\eta^{2}+\lambda^{2+2 \alpha} \xi^{2}+\lambda^{2 \alpha} \tau^{2}\right)^{-N_{0}} \frac{d \eta d \tau d \xi}{(2 \pi)^{3}} .
\end{aligned}
$$


By using the following estimates, for all $(\tau, \xi) \in[1,4]^{2}$ and $\lambda \geq \lambda_{0} \geq 1$,

$$
\begin{gathered}
1+\eta^{2}+\lambda^{2+2 \alpha} \xi^{2}+\lambda^{2 \alpha} \tau^{2} \leq c_{4} \lambda^{2+2 \alpha}\left(1+\eta^{2}\right), \\
\left(1+\eta^{2}+\lambda^{2+2 \alpha} \xi^{2}+\lambda^{2 \alpha} \tau^{2}\right)^{-N_{0}} \geq c_{4}^{-N_{0}} \lambda^{-2(1+\alpha) N_{0}}\left(1+\eta^{2}\right)^{-N_{0}},
\end{gathered}
$$

where $c_{4}=33$ and from (3.1.1), supp $\psi_{1} \subset[1,4]^{2}$ and $\psi_{1}=1$ on $[2,3]^{2}$, we deduce from (3.3.1) that for all $\lambda \geq \lambda_{0}$,

$$
\left\|u_{\lambda}\right\|_{\left(-N_{0}\right)}^{2} \geq \frac{c_{4}^{-N_{0}}}{(2 \pi)^{2}} \lambda^{-2(1+\alpha) N_{0}} \int_{[2,3]^{2}}\left\|g_{\lambda, \tau, \xi}\right\|_{H^{-N_{0}\left(\mathbb{R}_{y}\right)}}^{2} d \tau d \xi
$$

if we note

$$
g_{\lambda, \tau, \xi}(y)=\chi_{0}\left(\lambda^{\mu}\left(y+\left(\lambda^{-1} \xi^{-1} \tau\right)^{\frac{2}{k}}\right)\right) e^{-\Phi_{\lambda}(\tau, \xi, y)} .
$$

Then, we use that

$$
\left\|g_{\lambda, \tau, \xi}\right\|_{L^{2}\left(\mathbb{R}_{y}\right)}^{2} \leq\left\|g_{\lambda, \tau, \xi}\right\|_{H^{N_{0}\left(\mathbb{R}_{y}\right)}}\left\|g_{\lambda, \tau, \xi}\right\|_{H^{-N_{0}\left(\mathbb{R}_{y}\right)}} .
$$

The following lemma allows us to get an uniform lower bound, respectively to get an uniform upper bound with respect to the variables $(\tau, \xi)$ in $[2,3]^{2}$ for the quantities $\left\|g_{\lambda, \tau, \xi}\right\|_{L^{2}\left(\mathbb{R}_{y}\right)}$ and $\left\|g_{\lambda, \tau, \xi}\right\|_{H^{N_{0}\left(\mathbb{R}_{y}\right)}}$.

Lemma 3.3.1 We can find some positive constants $c_{5}$ and $c_{6}$ such that for all $(\tau, \xi) \in[2,3]^{2}$ and $\lambda \geq \lambda_{0}$,

$$
\left\|g_{\lambda, \tau, \xi}\right\|_{H^{N_{0}\left(\mathbb{R}_{y}\right)}} \leq c_{5} \lambda^{(\mu+2+2 \alpha) N_{0}-\frac{\mu}{2}} \text { and }\left\|g_{\lambda, \tau, \xi}\right\|_{L^{2}\left(\mathbb{R}_{y}\right)} \geq c_{6} \lambda^{-\frac{1+\alpha}{2}} .
$$

Proof. To obtain the first estimate, it is enough to get a bound for $\lambda \geq \lambda_{0}$ of the new quantities

$$
A_{j_{1}, j_{2}}(\lambda, \tau, \xi)=\left\|\lambda^{j_{1} \mu} \chi_{0}^{\left(j_{1}\right)}\left(\lambda^{\mu}\left(y+\left(\lambda^{-1} \xi^{-1} \tau\right)^{\frac{2}{k}}\right)\right) \partial_{y}^{j_{2}}\left(e^{-\Phi_{\lambda}(\tau, \xi, y)}\right)\right\|_{L^{2}\left(\mathbb{R}_{y}\right)},
$$

uniformly with respect to the variables $(\tau, \xi) \in[2,3]^{2}$ where $\left(j_{1}, j_{2}\right) \in \mathbb{N}^{2}$ are some integers verifying $j_{1}+j_{2}=N_{0}$. We obtain using (3.2.11) and the non-negativity of the function $\Phi_{\lambda}$ that for all $(\tau, \xi) \in[2,3]^{2}$ and $\lambda \geq \lambda_{0}$,

$$
\begin{aligned}
A_{j_{1}, j_{2}}(\lambda, \tau, \xi)^{2} & =\lambda^{2 j_{1} \mu} \int_{\mathbb{R}} \chi_{0}^{\left(j_{1}\right)}\left(\lambda^{\mu}\left(y+\left(\lambda^{-1} \xi^{-1} \tau\right)^{\frac{2}{k}}\right)\right)^{2}\left|\partial_{y}^{j_{2}}\left(e^{-\Phi_{\lambda}(\tau, \xi, y)}\right)\right|^{2} d y \\
& \leq C_{j_{2}}^{2} \lambda^{2 j_{1} \mu+4 j_{2}(1+\alpha)} \int_{\mathbb{R}} \chi_{0}^{\left(j_{1}\right)}\left(\lambda^{\mu}\left(y+\left(\lambda^{-1} \xi^{-1} \tau\right)^{\frac{2}{k}}\right)\right)^{2} d y \\
& =C_{j_{2}}^{2}\left\|\chi_{0}^{\left(j_{1}\right)}\right\|_{L^{2}(\mathbb{R})}^{2} \lambda^{2 j_{1} \mu+4 j_{2}(1+\alpha)-\mu} .
\end{aligned}
$$

We deduce from this last estimate that there exists a positive constant $c_{5}$ such that for all $(\tau, \xi) \in[2,3]^{2}$ and $\lambda \geq \lambda_{0}$,

$$
\left\|g_{\lambda, \tau, \xi}\right\|_{H^{N_{0}\left(\mathbb{R}_{y}\right)}} \leq c_{5} \lambda^{(\mu+2+2 \alpha) N_{0}-\frac{\mu}{2}},
$$


which shows the first estimate of (3.3.5). We want now to get an uniform lower bound for the quantity $\left\|g_{\lambda, \tau, \xi}\right\|_{L^{2}\left(\mathbb{R}_{y}\right)}$ with respect to the variables $(\tau, \xi) \in[2,3]^{2}$ for $\lambda \geq \lambda_{0}$. Using (3.1.10), we obtain that for all $y \in \mathbb{R}_{-}^{*}$ and $(\tau, \xi) \in \mathbb{R}_{+}^{*} \times \mathbb{R}_{+}^{*}$,

$$
\begin{aligned}
\Phi_{1}(\tau, \xi, y) & =\left(y+\left(\tau \xi^{-1}\right)^{\frac{2}{k}}\right)^{2} k \xi^{2} \int_{0}^{1}(1-\theta)\left(\left(\tau \xi^{-1}\right)^{\frac{2}{k}}(1-\theta)-\theta y\right)^{k-1} d \theta \\
& \leq k \xi^{2}\left(y+\left(\tau \xi^{-1}\right)^{\frac{2}{k}}\right)^{2}\left(\left(\tau \xi^{-1}\right)^{\frac{2}{k}}+|y|\right)^{k-1}
\end{aligned}
$$

We deduce from (3.1.11) and (3.3.6) that for all $y \in \mathbb{R}_{-}^{*},(\tau, \xi) \in[2,3]^{2}$ and $\lambda \geq 1$,

$$
\Phi_{\lambda}(\tau, \xi, y) \leq 9 k \lambda^{2+2 \alpha}\left(y+\left(\tau \xi^{-1} \lambda^{-1}\right)^{\frac{2}{k}}\right)^{2}\left(3^{\frac{2}{k}} 2^{-\frac{2}{k}}+|y|\right)^{k-1} .
$$

We obtain using (3.3.7) and the change of variables, $u=y+\left(\tau \xi^{-1} \lambda^{-1}\right)^{\frac{2}{k}}$, that for all $(\tau, \xi) \in[2,3]^{2}$ and $\lambda \geq 1$,

$$
\begin{aligned}
& \left\|e^{-\Phi_{\lambda}(\tau, \xi, y)} \mathbb{1}_{\mathbb{R}_{-}^{*}}(y)\right\|_{L^{2}\left(\mathbb{R}_{y}\right)}^{2} \\
\geq & \int_{-\infty}^{0} e^{-18 k \lambda^{2+2 \alpha}\left(y+\left(\tau \lambda^{-1} \xi^{-1}\right)^{\frac{2}{k}}\right)^{2}\left(3^{\frac{2}{k}} 2^{-\frac{2}{k}}+|y|\right)^{k-1}} d y \\
\geq & \int_{-\infty}^{-\left(\tau \xi^{-1} \lambda^{-1}\right)^{\frac{2}{k}}} e^{-18 k \lambda^{2+2 \alpha}\left(y+\left(\tau \lambda^{-1} \xi^{-1}\right)^{\frac{2}{k}}\right)^{2}\left(3^{\frac{2}{k}} 2^{-\frac{2}{k}}+|y|\right)^{k-1}} d y \\
\geq & \int_{-\infty}^{0} e^{-18 k \lambda^{2+2 \alpha} u^{2}\left(3^{\frac{2}{k}} 2^{-\frac{2}{k}}+\left|u-\left(\tau \xi^{-1} \lambda^{-1}\right)^{\frac{2}{k}}\right|\right)^{k-1}} d u .
\end{aligned}
$$

Since we can find a positive constant $c_{7}$ such that for all $y \in \mathbb{R}_{-}^{*},(\tau, \xi) \in$ $[2,3]^{2}$ and $\lambda \geq 1$,

$$
18 k\left(3^{\frac{2}{k}} 2^{-\frac{2}{k}}+\left|y-\left(\tau \xi^{-1} \lambda^{-1}\right)^{\frac{2}{k}}\right|\right)^{k-1} \leq c_{7}\left(1+|y|^{k-1}\right),
$$

it follows from (3.3.8) and (3.3.9),

$$
\left\|e^{-\Phi_{\lambda}(\tau, \xi, y)} \mathbb{1}_{\mathbb{R}_{-}^{*}}(y)\right\|_{L^{2}\left(\mathbb{R}_{y}\right)}^{2} \geq \int_{-\infty}^{0} e^{-c_{7} \lambda^{2+2 \alpha} u^{2}\left(1+|u|^{k-1}\right)} d u .
$$

Then using some changes of variables, we deduce from (3.3.10) that for all $(\tau, \xi) \in[2,3]^{2}$ and $\lambda \geq 1$,

$$
\begin{aligned}
& \left\|e^{-\Phi_{\lambda}(\tau, \xi, y)} \mathbb{1}_{\mathbb{R}_{-}^{*}}(y)\right\|_{L^{2}\left(\mathbb{R}_{y}\right)}^{2} \\
\geq & \int_{0}^{+\infty} e^{-c_{7} \lambda^{2+2 \alpha} u^{2}\left(1+u^{k-1}\right)} d u \\
= & \lambda^{-1-\alpha} \int_{0}^{+\infty} e^{-c_{7} v^{2}\left(1+v^{k-1} \lambda^{-(1+\alpha)(k-1)}\right)} d v \\
\geq & \lambda^{-1-\alpha} \int_{0}^{+\infty} e^{-c_{7} v^{2}\left(1+v^{k-1}\right)} d v=c_{8} \lambda^{-1-\alpha} .
\end{aligned}
$$


Next, if we note

$$
\tilde{\Omega}_{\lambda, \tau, \xi}=\left\{y \in \mathbb{R}_{-}^{*}:\left|y+\left(\lambda^{-1} \xi^{-1} \tau\right)^{\frac{2}{k}}\right| \geq 2^{-1} \lambda^{-\mu}\right\},
$$

using from (3.1.2) that $\chi_{0}=1$ on $[-1 / 2,1 / 2]$ and (3.2.16), we obtain that for all $(\tau, \xi) \in[2,3]^{2}$ and $\lambda \geq 1$,

$$
\begin{aligned}
& \left\|\left[1-\chi_{0}\left(\lambda^{\mu}\left(y+\left(\lambda^{-1} \xi^{-1} \tau\right)^{\frac{2}{k}}\right)\right)\right] e^{-\Phi_{\lambda}(\tau, \xi, y)} \mathbb{1}_{\mathbb{R}_{-}^{*}}(y)\right\|_{L^{2}\left(\mathbb{R}_{y}\right)}^{2} \\
\leq & \left\|1-\chi_{0}\right\|_{L^{\infty}(\mathbb{R})}^{2} \int_{\tilde{\Omega}_{\lambda, \tau, \xi}} e^{-2 \Phi_{\lambda}(\tau, \xi, y)} d y \\
\leq & \left\|1-\chi_{0}\right\|_{L^{\infty}(\mathbb{R})}^{2} \int_{\tilde{\Omega}_{\lambda, \tau, \xi}} e^{-2 c_{3} \lambda^{2 \alpha+\frac{2}{k}}\left(y+\left(\lambda^{-1} \xi^{-1} \tau\right)^{\frac{2}{k}}\right)^{2}} d y \\
\leq & e^{-\frac{c_{3}}{4} \lambda^{2 \alpha+\frac{2}{k}-2 \mu}}\left\|1-\chi_{0}\right\|_{L^{\infty}(\mathbb{R})}^{2} \int_{\mathbb{R}} e^{-c_{3} \lambda^{2 \alpha+\frac{2}{k}}\left(y+\left(\lambda^{-1} \xi^{-1} \tau\right)^{\frac{2}{k}}\right)^{2}} d y \\
\leq & \pi^{\frac{1}{2}} c_{3}^{-\frac{1}{2}}\left\|1-\chi_{0}\right\|_{L^{\infty}(\mathbb{R})}^{2} \lambda^{-\alpha-\frac{1}{k}} e^{-\frac{c_{3}}{4} \lambda^{2\left(\alpha+\frac{1}{k}-\mu\right)}},
\end{aligned}
$$

since if $y \in \tilde{\Omega}_{\lambda, \tau, \xi}$, we have

$$
e^{-2 c_{3} \lambda^{2 \alpha+\frac{2}{k}}\left(y+\left(\lambda^{-1} \xi^{-1} \tau\right)^{\frac{2}{k}}\right)^{2}} \leq e^{-\frac{c_{3}}{4} \lambda^{2 \alpha+\frac{2}{k}-2 \mu}} e^{-c_{3} \lambda^{2 \alpha+\frac{2}{k}}\left(y+\left(\lambda^{-1} \xi^{-1} \tau\right)^{\frac{2}{k}}\right)^{2}}
$$

and since a change of variables gives that

$$
\int_{\mathbb{R}} e^{-c_{3} \lambda^{2 \alpha+\frac{2}{k}}\left(y+\left(\lambda^{-1} \xi^{-1} \tau\right)^{\frac{2}{k}}\right)^{2}} d y=\int_{\mathbb{R}} e^{-c_{3} \lambda^{2 \alpha+\frac{2}{k}} y^{2}} d y=\pi^{\frac{1}{2}} c_{3}^{-\frac{1}{2}} \lambda^{-\alpha-\frac{1}{k}} .
$$

In view of (3.1.5) and (3.3.3), the use of the triangular inequality for all $(\tau, \xi) \in[2,3]^{2}$ and $\lambda \geq \lambda_{0}$,

$$
\begin{aligned}
\left\|g_{\lambda, \tau, \xi}\right\|_{L^{2}\left(\mathbb{R}_{y}\right)} & =\left\|\chi_{0}\left(\lambda^{\mu}\left(y+\left(\lambda^{-1} \xi^{-1} \tau\right)^{\frac{2}{k}}\right)\right) e^{-\Phi_{\lambda}(\tau, \xi, y)} \mathbb{1}_{\mathbb{R}_{-}^{*}}(y)\right\|_{L^{2}\left(\mathbb{R}_{y}\right)} \\
& \geq\left\|e^{-\Phi_{\lambda}(\tau, \xi, y)} \mathbb{1}_{\mathbb{R}_{-}^{*}}(y)\right\|_{L^{2}\left(\mathbb{R}_{y}\right)} \\
& -\left\|\left[1-\chi_{0}\left(\lambda^{\mu}\left(y+\left(\lambda^{-1} \xi^{-1} \tau\right)^{\frac{2}{k}}\right)\right)\right] e^{-\Phi_{\lambda}(\tau, \xi, y)} \mathbb{1}_{\mathbb{R}_{-}^{*}}(y)\right\|_{L^{2}\left(\mathbb{R}_{y}\right)},
\end{aligned}
$$

with the estimates (3.3.11) and (3.3.13), shows that there exists a positive constant $c_{6}$ such that for all $(\tau, \xi) \in[2,3]^{2}$ and $\lambda \geq \lambda_{0}$,

$$
\left\|g_{\lambda, \tau, \xi}\right\|_{L^{2}\left(\mathbb{R}_{y}\right)} \geq c_{6} \lambda^{-\frac{1+\alpha}{2}}
$$

because from (3.1.3),

$$
\alpha+\frac{1}{k}-\mu>0 .
$$

This ends the proof of Lemma 3.3.1. 
The previous lemma permits us to obtain from the estimate (3.3.4) that for all $(\tau, \xi) \in[2,3]^{2}$ and $\lambda \geq \lambda_{0}$,

$$
\left\|g_{\lambda, \tau, \xi}\right\|_{H^{-N_{0}\left(\mathbb{R}_{y}\right)}} \geq c_{6}^{2} c_{5}^{-1} \lambda^{-\alpha-1+\frac{\mu}{2}-(\mu+2+2 \alpha) N_{0}} .
$$

Then using (3.3.2), we obtain the following proposition.

Proposition 3.3.1 There exists a positive constant $c_{9}$ such that for all $\lambda \geq$ $\lambda_{0}$,

$$
\left\|u_{\lambda}\right\|_{\left(-N_{0}\right)} \geq c_{9} \lambda^{-\alpha-1+\frac{\mu}{2}-(\mu+3+3 \alpha) N_{0}} .
$$

We need now to cutoff in the variables $t, x$ to obtain a quasimode localized in an arbitrary neighbourhood of 0 in $\mathbb{R}^{3}$.

\subsection{Cutoff in variables $t$ and $x$}

We need first to make the result of the lemma 3.2.1 more precise when there is no differentiation in the variable $y$.

Lemma 3.4.1 For all $\rho \in \mathbb{N}^{2}$, there exist some functions $a_{l}, l=0, \ldots,|\rho|(k+$ $1)$, which are polynomial in $\mathbb{R}^{4}$ and some constants $\beta_{l}, l=0, \ldots,|\rho|(k+1)$ verifying

$$
\beta_{l} \leq 2|\rho|\left(\alpha-\frac{1}{k}\right)
$$

such that for all $y \in \mathbb{R}_{-}^{*},(\tau, \xi) \in \mathbb{R}_{+}^{*} \times \mathbb{R}_{+}^{*}$ and $\lambda \geq 1$,

$$
\partial_{\tau, \xi}^{\rho}\left(e^{-\Phi_{\lambda}}\right)=e^{-\Phi_{\lambda}} \sum_{l=0}^{|\rho|(k+1)} a_{l}\left(\tau^{\frac{1}{k}}, \tau^{-\frac{1}{k}}, \xi^{\frac{1}{k}}, \xi^{-\frac{1}{k}}\right)\left(\lambda^{\frac{2}{k}} y\right)^{l} \lambda^{\beta_{l}} .
$$

Proof. We prove again this lemma by induction on $|\rho|$. If $|\rho|=0$, the expression (3.4.1) holds with $a_{0}=1$ and $\beta_{0}=0$. Let us assume now that for $\rho \in \mathbb{N}^{2}$, there exist some functions $a_{l}, l=0, \ldots,|\rho|(k+1)$, which are polynomial in $\mathbb{R}^{4}$ and some constants $\beta_{l}, l=0, \ldots,|\rho|(k+1)$ verifying $\beta_{l} \leq 2|\rho|(\alpha-1 / k)$ such that for all $y \in \mathbb{R}_{-}^{*},(\tau, \xi) \in \mathbb{R}_{+}^{*} \times \mathbb{R}_{+}^{*}, \lambda \geq 1$, the expression (3.4.1) holds. Since we can write (3.2.5) and (3.2.6) in the following way,

$$
\begin{gathered}
\partial_{\tau} \Phi_{\lambda}(\tau, \xi, y)=\lambda^{2 \alpha-\frac{2}{k}}\left(2 \tau\left(\lambda^{\frac{2}{k}} y\right)+2 \xi^{-\frac{2}{k}} \tau^{\frac{k+2}{k}}\right), \\
\partial_{\xi} \Phi_{\lambda}(\tau, \xi, y)=\frac{2}{k+1} \lambda^{2 \alpha-\frac{2}{k}}\left(-\tau^{\frac{2 k+2}{k}} \xi^{-\frac{k+2}{k}}+(-1)^{k+1} \xi\left(\lambda^{\frac{2}{k}} y\right)^{k+1}\right)
\end{gathered}
$$


and

$$
\begin{aligned}
& \partial_{\tau}\left(e^{-\Phi_{\lambda}} \sum_{l=0}^{|\rho|(k+1)} a_{l}\left(\tau^{\frac{1}{k}}, \tau^{-\frac{1}{k}}, \xi^{\frac{1}{k}}, \xi^{-\frac{1}{k}}\right)\left(\lambda^{\frac{2}{k}} y\right)^{l} \lambda^{\beta_{l}}\right) \\
& =e^{-\Phi_{\lambda}}\left(\sum _ { l = 0 } ^ { | \rho | ( k + 1 ) } \left[-\left(\partial_{\tau} \Phi_{\lambda}\right) a_{l}\left(\tau^{\frac{1}{k}}, \tau^{-\frac{1}{k}}, \xi^{\frac{1}{k}}, \xi^{-\frac{1}{k}}\right)\right.\right. \\
& \left.\left.+\partial_{\tau}\left(a_{l}\left(\tau^{\frac{1}{k}}, \tau^{-\frac{1}{k}}, \xi^{\frac{1}{k}}, \xi^{-\frac{1}{k}}\right)\right)\right]\left(\lambda^{\frac{2}{k}} y\right)^{l} \lambda^{\beta_{l}}\right), \\
& \partial_{\xi}\left(e^{-\Phi_{\lambda}} \sum_{l=0}^{|\rho|(k+1)} a_{l}\left(\tau^{\frac{1}{k}}, \tau^{-\frac{1}{k}}, \xi^{\frac{1}{k}}, \xi^{-\frac{1}{k}}\right)\left(\lambda^{\frac{2}{k}} y\right)^{l} \lambda^{\beta_{l}}\right) \\
& =e^{-\Phi_{\lambda}}\left(\sum _ { l = 0 } ^ { | \rho | ( k + 1 ) } \left[-\left(\partial_{\xi} \Phi_{\lambda}\right) a_{l}\left(\tau^{\frac{1}{k}}, \tau^{-\frac{1}{k}}, \xi^{\frac{1}{k}}, \xi^{-\frac{1}{k}}\right)\right.\right. \\
& \left.\left.+\partial_{\xi}\left(a_{l}\left(\tau^{\frac{1}{k}}, \tau^{-\frac{1}{k}}, \xi^{\frac{1}{k}}, \xi^{-\frac{1}{k}}\right)\right)\right]\left(\lambda^{\frac{2}{k}} y\right)^{l} \lambda^{\beta_{l}}\right),
\end{aligned}
$$

we obtain that

$$
\begin{gathered}
\partial_{\tau}\left(e^{-\Phi_{\lambda}} \sum_{l=0}^{|\rho|(k+1)} a_{l}\left(\tau^{\frac{1}{k}}, \tau^{-\frac{1}{k}}, \xi^{\frac{1}{k}}, \xi^{-\frac{1}{k}}\right)\left(\lambda^{\frac{2}{k}} y\right)^{l} \lambda^{\beta_{l}}\right)= \\
-e^{-\Phi_{\lambda}} \sum_{l=0}^{|\rho|(k+1)} 2\left(\tau^{\frac{1}{k}}\right)^{k} a_{l}\left(\tau^{\frac{1}{k}}, \tau^{-\frac{1}{k}}, \xi^{\frac{1}{k}}, \xi^{-\frac{1}{k}}\right)\left(\lambda^{\frac{2}{k}} y\right)^{l+1} \lambda^{\beta_{l}+2\left(\alpha-\frac{1}{k}\right)} \\
-e^{-\Phi_{\lambda}} \sum_{l=0}^{|\rho|(k+1)} 2\left(\xi^{-\frac{1}{k}}\right)^{2}\left(\tau^{\frac{1}{k}}\right)^{k+2} a_{l}\left(\tau^{\frac{1}{k}}, \tau^{-\frac{1}{k}}, \xi^{\frac{1}{k}}, \xi^{-\frac{1}{k}}\right)\left(\lambda^{\frac{2}{k}} y\right)^{l} \lambda^{\beta_{l}+2\left(\alpha-\frac{1}{k}\right)} \\
+e^{-\Phi_{\lambda}} \sum_{l=0}^{|\rho|(k+1)}\left(\frac { 1 } { k } \left[\tau^{\frac{1}{k}}\left(\tau^{-\frac{1}{k}}\right)^{k}\left(\partial_{1} a_{l}\right)\left(\tau^{\frac{1}{k}}, \tau^{-\frac{1}{k}}, \xi^{\frac{1}{k}}, \xi^{-\frac{1}{k}}\right)\right.\right. \\
\left.\left.-\left(\tau^{-\frac{1}{k}}\right)^{k+1}\left(\partial_{2} a_{l}\right)\left(\tau^{\frac{1}{k}}, \tau^{-\frac{1}{k}}, \xi^{\frac{1}{k}}, \xi^{-\frac{1}{k}}\right)\right]\left(\lambda^{\frac{2}{k}} y\right)^{l} \lambda^{\beta_{l}}\right)
\end{gathered}
$$


and

$$
\begin{gathered}
\partial_{\xi}\left(e^{-\Phi_{\lambda}} \sum_{l=0}^{|\rho|(k+1)} a_{l}\left(\tau^{\frac{1}{k}}, \tau^{-\frac{1}{k}}, \xi^{\frac{1}{k}}, \xi^{-\frac{1}{k}}\right)\left(\lambda^{\frac{2}{k}} y\right)^{l} \lambda^{\beta_{l}}\right)= \\
e^{-\Phi_{\lambda}} \sum_{l=0}^{|\rho|(k+1)} \frac{2}{k+1}\left(\tau^{\frac{1}{k}}\right)^{2 k+2}\left(\xi^{-\frac{1}{k}}\right)^{k+2} a_{l}\left(\tau^{\frac{1}{k}}, \tau^{-\frac{1}{k}}, \xi^{\frac{1}{k}}, \xi^{-\frac{1}{k}}\right)\left(\lambda^{\frac{2}{k}} y\right)^{l} \lambda^{\beta_{l}+2\left(\alpha-\frac{1}{k}\right)} \\
-e^{-\Phi_{\lambda}} \sum_{l=0}^{|\rho|(k+1)} \frac{2}{k+1}(-1)^{k+1}\left(\xi^{\frac{1}{k}}\right)^{k} a_{l}\left(\tau^{\frac{1}{k}}, \tau^{-\frac{1}{k}}, \xi^{\frac{1}{k}}, \xi^{-\frac{1}{k}}\right)\left(\lambda^{\frac{2}{k}} y\right)^{l+k+1} \lambda^{\beta_{l}+2\left(\alpha-\frac{1}{k}\right)} \\
\sum_{l=0}^{-\Phi_{\lambda}}\left(\frac { 1 } { k } \left[\xi^{\frac{1}{k}}\left(\xi^{-\frac{1}{k}}\right)^{k}\left(\partial_{3} a_{l}\right)\left(\tau^{\frac{1}{k}}, \tau^{-\frac{1}{k}}, \xi^{\frac{1}{k}}, \xi^{-\frac{1}{k}}\right)\right.\right. \\
\left.\left.-\left(\xi^{-\frac{1}{k}}\right)^{k+1}\left(\partial_{4} a_{l}\right)\left(\tau^{\frac{1}{k}}, \tau^{-\frac{1}{k}}, \xi^{\frac{1}{k}}, \xi^{-\frac{1}{k}}\right)\right]\left(\lambda^{\frac{2}{k}} y\right)^{l} \lambda^{\beta_{l}}\right) .
\end{gathered}
$$

Since from (3.1.3), $\alpha>1 / k$, we deduce from (3.4.6) and (3.4.7) that if $\tilde{\rho} \in\{\rho+(1,0), \rho+(0,1)\}$, there exist some functions $\tilde{a}_{l}, l=0, \ldots,|\tilde{\rho}|(k+1)$, which are polynomial in $\mathbb{R}^{4}$ and some constants $\tilde{\beta}_{l}, l=0, \ldots,|\tilde{\rho}|(k+1)$ verifying $\tilde{\beta}_{l} \leq 2|\tilde{\rho}|(\alpha-1 / k)$ such that for all $y \in \mathbb{R}_{-}^{*},(\tau, \xi) \in \mathbb{R}_{+}^{*} \times \mathbb{R}_{+}^{*}$ and $\lambda \geq 1$,

$$
\partial_{\tau, \xi}^{\tilde{\rho}}\left(e^{-\Phi_{\lambda}}\right)=e^{-\Phi_{\lambda}} \sum_{l=0}^{|\tilde{\rho}|(k+1)} \tilde{a}_{l}\left(\tau^{\frac{1}{k}}, \tau^{-\frac{1}{k}}, \xi^{\frac{1}{k}}, \xi^{-\frac{1}{k}}\right)\left(\lambda^{\frac{2}{k}} y\right)^{l} \lambda^{\tilde{\beta}_{l}} .
$$

This proves the induction property at the rank $|\rho|+1$ and ends the proof of the lemma 3.4.1.

We can now prove the following lemma.

Lemma 3.4.2 For all $\rho \in \mathbb{N}^{2}$, there exists a positive constant $M_{\rho}$ such that for all $(\tau, \xi) \in[1,4]^{2}, \lambda \geq \lambda_{0}$ and $y \in \operatorname{supp} \chi_{0}\left(\lambda^{\mu}\left(\cdot+\left(\lambda^{-1} \xi^{-1} \tau\right)^{\frac{2}{k}}\right)\right)$,

$$
\left|\partial_{\tau, \xi}^{\rho}\left(e^{-\Phi_{\lambda}(\tau, \xi, y)}\right)\right| \leq M_{\rho} \lambda^{2|\rho|\left(\alpha-\frac{1}{k}\right)} .
$$

Proof. We recall that the above notation supp $\chi_{0}\left(\lambda^{\mu}\left(\cdot+\left(\lambda^{-1} \xi^{-1} \tau\right)^{\frac{2}{k}}\right)\right)$ stands for the support of the function

$$
y \mapsto \chi_{0}\left(\lambda^{\mu}\left(y+\left(\lambda^{-1} \xi^{-1} \tau\right)^{\frac{2}{k}}\right)\right) .
$$

It follows from the previous lemma that there exist some functions $a_{l}, l=$ $0, \ldots,|\rho|(k+1)$, which are polynomial in $\mathbb{R}^{4}$ and some constants $\beta_{l}, l=$ $0, \ldots,|\rho|(k+1)$ verifying

$$
\beta_{l} \leq 2|\rho|\left(\alpha-\frac{1}{k}\right)
$$


such that for all $y \in \mathbb{R}_{-}^{*},(\tau, \xi) \in \mathbb{R}_{+}^{*} \times \mathbb{R}_{+}^{*}$ and $\lambda \geq 1$,

$$
\partial_{\tau, \xi}^{\rho}\left(e^{-\Phi_{\lambda}}\right)=e^{-\Phi_{\lambda}} \sum_{l=0}^{|\rho|(k+1)} a_{l}\left(\tau^{\frac{1}{k}}, \tau^{-\frac{1}{k}}, \xi^{\frac{1}{k}}, \xi^{-\frac{1}{k}}\right)\left(\lambda^{\frac{2}{k}} y\right)^{l} \lambda^{\beta_{l}} .
$$

Using the non-negativity of the phase function $\Phi_{\lambda}$ (see (3.1.10) and (3.1.11)), we deduce from (3.1.5) and (3.4.9) that for $l=0, \ldots,|\rho|(k+1)$, there exists a positive constant $c_{10, l}$ such that for all $(\tau, \xi) \in[1,4]^{2}, \lambda \geq \lambda_{0}$ and

$$
\begin{gathered}
y \in \operatorname{supp} \chi_{0}\left(\lambda^{\mu}\left(\cdot+\left(\lambda^{-1} \xi^{-1} \tau\right)^{\frac{2}{k}}\right)\right), \\
\left|e^{-\Phi_{\lambda}} a_{l}\left(\tau^{\frac{1}{k}}, \tau^{-\frac{1}{k}}, \xi^{\frac{1}{k}}, \xi^{-\frac{1}{k}}\right) \lambda^{\beta_{l}}\right| \leq c_{10, l} \lambda^{2|\rho|\left(\alpha-\frac{1}{k}\right)},
\end{gathered}
$$

since from (3.1.3), $\alpha>1 / k$. Finally, in view of (3.1.5), (3.4.10) and (3.4.11), we deduce that there exists a positive constant $M_{\rho}$ such that the estimate (3.4.8) holds. This ends the proof of the lemma 3.4.2.

Let us now consider the function $v_{\lambda}$ defined by

$$
v_{\lambda}(t, x, y)=\chi_{1}\left(\lambda^{\gamma} t, \lambda^{\gamma} x\right) u_{\lambda}(t, x, y),
$$

where $\gamma$ is a parameter verifying

$$
0<\gamma<\frac{1}{k} \text { and } \gamma+\alpha<\frac{2}{k}
$$

This choice is possible in view of (3.1.3). The function $\chi_{1}$ is taken in the space $C_{0}^{\infty}\left(\mathbb{R}^{2}, \mathbb{R}\right)$ such that

$$
\text { supp } \chi_{1} \subset B(0,1) \text { and } \chi_{1}=1 \text { on } B(0,1 / 2) \text {, }
$$

where the notation $B(0, r)$ stands for the closed Euclidean ball centered in 0 with a radius $r$. We start by getting a lower bound for the quantity $\left\|v_{\lambda}\right\|_{\left(-N_{0}\right)}$. To do this, we prove the following lemma.

Lemma 3.4.3 For all $M \in \mathbb{N}$, there exists a positive constant $K_{M}$ such that for all $\lambda \geq \lambda_{0}$,

$$
\left\|\left(1-\chi_{1}\left(\lambda^{\gamma} t, \lambda^{\gamma} x\right)\right) u_{\lambda}(t, x, y)\right\|_{\left(-N_{0}\right)} \leq K_{M} \lambda^{-M} .
$$

Proof. Since from (3.1.4), (3.1.11), (3.1.13) and a change of variables

$$
\begin{aligned}
& u_{\lambda}(t, x, y)=\frac{\lambda^{\frac{1}{2}+\alpha}}{(2 \pi)^{2}} \\
& \times \int_{\mathbb{R}^{2}} e^{i\left(x \xi \lambda^{1+\alpha}+t \tau \lambda^{\alpha}\right)} \psi_{1}(\tau, \xi) \chi_{0}\left(\lambda^{\mu}\left(y+\left(\tau \xi^{-1} \lambda^{-1}\right)^{\frac{2}{k}}\right)\right) e^{-\Phi_{\lambda}(\tau, \xi, y)} d \tau d \xi
\end{aligned}
$$


we deduce from (3.1.1), (3.1.5) and (3.4.14) that for all $\lambda \geq \lambda_{0}$,

$$
\begin{aligned}
& \left\|\left(1-\chi_{1}\left(\lambda^{\gamma} t, \lambda^{\gamma} x\right)\right) u_{\lambda}(t, x, y)\right\|_{\left(-N_{0}\right)} \\
\leq & \left\|\left(1-\chi_{1}\left(\lambda^{\gamma} t, \lambda^{\gamma} x\right)\right) u_{\lambda}(t, x, y)\right\|_{L^{2}\left(\mathbb{R}^{3}\right)} \\
\leq & \left\|1-\chi_{1}\right\|_{L^{\infty}\left(\mathbb{R}^{2}\right)} \\
& \times\left(\int_{-c_{1} \lambda^{-\frac{2}{k}}}^{-c_{2} \lambda^{-\frac{2}{k}}}\left[\int_{\left\{(t, x) \in \mathbb{R}^{2}: t^{2}+x^{2} \geq 4^{-1} \lambda^{-2 \gamma}\right\}}\left|u_{\lambda}(t, x, y)\right|^{2} d t d x\right] d y\right)^{\frac{1}{2}} .
\end{aligned}
$$

Now, some integrations by parts on (3.4.16) show that for all $q \in \mathbb{N},(t, x) \neq$ $(0,0), y \in \mathbb{R}$ and $\lambda \geq \lambda_{0}$,

$$
\begin{aligned}
u_{\lambda}(t, x, y)= & \frac{\lambda^{\alpha+\frac{1}{2}}\left(i \lambda^{1+\alpha} x+\lambda^{\alpha} t\right)^{-q}}{(2 \pi)^{2}} \int_{\mathbb{R}^{2}}\left(\partial_{\xi}-i \partial_{\tau}\right)^{q}\left(e^{i\left(\lambda^{1+\alpha} x \xi+\lambda^{\alpha} t \tau\right)}\right) \\
& \times \psi_{1}(\tau, \xi) \chi_{0}\left(\lambda^{\mu}\left(y+\left(\lambda^{-1} \xi^{-1} \tau\right)^{\frac{2}{k}}\right)\right) e^{-\Phi_{\lambda}(\tau, \xi, y)} d \tau d \xi \\
= & \frac{\lambda^{\alpha+\frac{1}{2}}\left(i \lambda^{1+\alpha} x+\lambda^{\alpha} t\right)^{-q}}{(2 \pi)^{2}} \int_{\mathbb{R}^{2}} e^{i\left(\lambda^{1+\alpha} x \xi+\lambda^{\alpha} t \tau\right)}\left(i \partial_{\tau}-\partial_{\xi}\right)^{q} \\
& {\left[\chi_{0}\left(\lambda^{\mu}\left(y+\left(\lambda^{-1} \xi^{-1} \tau\right)^{\frac{2}{k}}\right)\right) \psi_{1}(\tau, \xi) e^{-\Phi_{\lambda}(\tau, \xi, y)}\right] d \tau d \xi }
\end{aligned}
$$

We need the following lemma.

Lemma 3.4.4 For all $\rho \in \mathbb{N}^{2}$ and $l \in \mathbb{N}$, there exists a positive constant $c_{11, \rho, l}$ such that for all $y \in \mathbb{R},(\tau, \xi) \in[1,4]^{2}$ and $\lambda \geq 1$,

$$
\left|\partial_{\tau, \xi}^{\rho}\left[\chi_{0}^{(l)}\left(\lambda^{\mu}\left(y+\left(\lambda^{-1} \xi^{-1} \tau\right)^{\frac{2}{k}}\right)\right)\right]\right| \leq c_{11, \rho, l} \lambda^{|\rho|\left(\mu-\frac{2}{k}\right)} .
$$

Proof of the lemma 3.4.4. We start by proving that for all $\rho \in \mathbb{N}^{2}$ and $l \in \mathbb{N}$, there exist some polynomial functions $P_{\rho, l, j}$ in $\mathbb{R}^{4}, j=0, \ldots,|\rho|$, such that for all $y \in \mathbb{R},(\tau, \xi) \in \mathbb{R}_{+}^{*} \times \mathbb{R}_{+}^{*}$ and $\lambda \geq 1$,

$$
\begin{aligned}
& \partial_{\tau, \xi}^{\rho}\left[\chi_{0}^{(l)}\left(\lambda^{\mu}\left(y+\left(\lambda^{-1} \xi^{-1} \tau\right)^{\frac{2}{k}}\right)\right)\right]= \\
& \sum_{j=0}^{|\rho|} P_{\rho, l, j}\left(\tau^{\frac{1}{k}}, \tau^{-\frac{1}{k}}, \xi^{\frac{1}{k}}, \xi^{-\frac{1}{k}}\right) \chi_{0}^{(l+j)}\left(\lambda^{\mu}\left(y+\left(\lambda^{-1} \xi^{-1} \tau\right)^{\frac{2}{k}}\right)\right) \lambda^{j\left(\mu-\frac{2}{k}\right)}
\end{aligned}
$$

Let us consider $l \in \mathbb{N}$. We prove (3.4.20) by induction on $|\rho|$. If $|\rho|=0$, the identity (3.4.20) holds with $P_{\rho, l, 0}=1$. Let us assume now that the identity 
(3.4.20) holds for $\rho \in \mathbb{N}^{2}$. The two direct computations using (3.4.20),

$$
\begin{gathered}
\partial_{\tau, \xi}^{\tilde{\rho}}\left[\chi_{0}^{(l)}\left(\lambda^{\mu}\left(y+\left(\lambda^{-1} \xi^{-1} \tau\right)^{\frac{2}{k}}\right)\right)\right] \\
=\sum_{j=0}^{|\tilde{\rho}|-1}\left[\frac{2}{k}\left(\xi^{-\frac{1}{k}}\right)^{2}\left(\tau^{\frac{1}{k}}\right)^{2-k} P_{\rho, l, j}\left(\tau^{\frac{1}{k}}, \tau^{-\frac{1}{k}}, \xi^{\frac{1}{k}}, \xi^{-\frac{1}{k}}\right)\right. \\
\left.\times \chi_{0}^{(l+j+1)}\left(\lambda^{\mu}\left(y+\left(\lambda^{-1} \xi^{-1} \tau\right)^{\frac{2}{k}}\right)\right) \lambda^{(j+1)\left(\mu-\frac{2}{k}\right)}\right] \\
+\sum_{j=0}^{|\tilde{\rho}|-1}\left[\frac{1}{k}\left(\tau^{\frac{1}{k}}\right)^{1-k}\left(\partial_{1} P_{\rho, l, j}\right)\left(\tau^{\frac{1}{k}}, \tau^{-\frac{1}{k}}, \xi^{\frac{1}{k}}, \xi^{-\frac{1}{k}}\right)\right. \\
\left.\times \chi_{0}^{(l+j)}\left(\lambda^{\mu}\left(y+\left(\lambda^{-1} \xi^{-1} \tau\right)^{\frac{2}{k}}\right)\right) \lambda^{j\left(\mu-\frac{2}{k}\right)}\right] \\
-\sum_{j=0}^{|\tilde{\rho}|-1}\left[\frac{1}{k}\left(\tau^{-\frac{1}{k}}\right)^{k+1}\left(\partial_{2} P_{\rho, l, j}\right)\left(\tau^{\frac{1}{k}}, \tau^{-\frac{1}{k}}, \xi^{\frac{1}{k}}, \xi^{-\frac{1}{k}}\right)\right. \\
\left.\times \chi_{0}^{(l+j)}\left(\lambda^{\mu}\left(y+\left(\lambda^{-1} \xi^{-1} \tau\right)^{\frac{2}{k}}\right)\right) \lambda^{j\left(\mu-\frac{2}{k}\right)}\right],
\end{gathered}
$$

if $\tilde{\rho}=\rho+(1,0)$ and

$$
\begin{gathered}
\partial_{\tau, \xi}^{\tilde{\rho}}\left[\chi_{0}^{(l)}\left(\lambda^{\mu}\left(y+\left(\lambda^{-1} \xi^{-1} \tau\right)^{\frac{2}{k}}\right)\right)\right]= \\
-\sum_{j=0}^{|\tilde{\rho}|-1}\left[\frac{2}{k}\left(\xi^{-\frac{1}{k}}\right)^{k+2}\left(\tau^{\frac{1}{k}}\right)^{2} P_{\rho, l, j}\left(\tau^{\frac{1}{k}}, \tau^{-\frac{1}{k}}, \xi^{\frac{1}{k}}, \xi^{-\frac{1}{k}}\right)\right. \\
\left.\times \chi_{0}^{(l+j+1)}\left(\lambda^{\mu}\left(y+\left(\lambda^{-1} \xi^{-1} \tau\right)^{\frac{2}{k}}\right)\right) \lambda^{(j+1)\left(\mu-\frac{2}{k}\right)}\right] \\
+\sum_{j=0}^{|\tilde{\rho}|-1}\left[\frac{1}{k}\left(\xi^{\frac{1}{k}}\right)^{1-k}\left(\partial_{3} P_{\rho, l, j}\right)\left(\tau^{\frac{1}{k}}, \tau^{-\frac{1}{k}}, \xi^{\frac{1}{k}}, \xi^{-\frac{1}{k}}\right)\right. \\
\left.\times \chi_{0}^{(l+j)}\left(\lambda^{\mu}\left(y+\left(\lambda^{-1} \xi^{-1} \tau\right)^{\frac{2}{k}}\right)\right) \lambda^{j\left(\mu-\frac{2}{k}\right)}\right] \\
-\sum_{j=0}^{|\tilde{\rho}|-1}\left[\frac{1}{k}\left(\xi^{-\frac{1}{k}}\right)^{k+1}\left(\partial_{4} P_{\rho, l, j}\right)\left(\tau^{\frac{1}{k}}, \tau^{-\frac{1}{k}}, \xi^{\frac{1}{k}}, \xi^{-\frac{1}{k}}\right)\right. \\
\left.\times \chi_{0}^{(l+j)}\left(\lambda^{\mu}\left(y+\left(\lambda^{-1} \xi^{-1} \tau\right)^{\frac{2}{k}}\right)\right) \lambda^{j\left(\mu-\frac{2}{k}\right)}\right],
\end{gathered}
$$

if $\tilde{\rho}=\rho+(0,1)$, prove that the induction property holds at the rank $|\rho|+1$. This proves (3.4.20). Since from (3.1.2), $\chi_{0} \in C_{0}^{\infty}(\mathbb{R}, \mathbb{R})$ and from (3.1.3), $\mu>2 / k$, we deduce from (3.4.20) that there exists a positive constant $c_{11, \rho, l}$ such that for all $y \in \mathbb{R},(\tau, \xi) \in[1,4]^{2}$ and $\lambda \geq 1$,

$$
\left|\partial_{\tau, \xi}^{\rho}\left[\chi_{0}^{(l)}\left(\lambda^{\mu}\left(y+\left(\lambda^{-1} \xi^{-1} \tau\right)^{\frac{2}{k}}\right)\right)\right]\right| \leq c_{11, \rho, l} \lambda^{|\rho|\left(\mu-\frac{2}{k}\right)},
$$

which ends the proof of the lemma 3.4.4. 
Then, we obtain using the lemma 3.4.2, (3.1.1), (3.4.19) and the Leibniz formula on the expression

$$
\left(i \partial_{\tau}-\partial_{\xi}\right)^{q}\left[\chi_{0}\left(\lambda^{\mu}\left(y+\left(\lambda^{-1} \xi^{-1} \tau\right)^{\frac{2}{k}}\right)\right) \psi_{1}(\tau, \xi) e^{-\Phi_{\lambda}(\tau, \xi, y)}\right],
$$

where $q \in \mathbb{N}$, that there exist some positive constants $c_{12, j}, j=0, \ldots, q$ and $c_{13}$ such that for all $y \in \mathbb{R},(\tau, \xi) \in \mathbb{R}^{2}$ and $\lambda \geq \lambda_{0}$,

$$
\begin{aligned}
& \left|\left(i \partial_{\tau}-\partial_{\xi}\right)^{q}\left[\chi_{0}\left(\lambda^{\mu}\left(y+\left(\lambda^{-1} \xi^{-1} \tau\right)^{\frac{2}{k}}\right)\right) \psi_{1}(\tau, \xi) e^{-\Phi_{\lambda}(\tau, \xi, y)}\right]\right| \\
\leq & \sum_{j=0}^{q} c_{12, j} \lambda^{\left(\mu-\frac{2}{k}\right) j} \lambda^{2(q-j)\left(\alpha-\frac{1}{k}\right)} \\
\leq & c_{13} \lambda^{q \max \left(\mu-\frac{2}{k}, 2 \alpha-\frac{2}{k}\right)}
\end{aligned}
$$

since from (3.1.3),

$$
\mu>\frac{2}{k} \text { and } \alpha>\frac{1}{k} \text {. }
$$

Since from (3.1.1), supp $\psi_{1} \subset[1,4]^{2}$, it follows from (3.4.18) and (3.4.21) that for all $q \in \mathbb{N}$, there exists a positive constant $c_{14, q}$ such that for all $(t, x) \neq(0,0), y \in \mathbb{R}$ and $\lambda \geq \lambda_{0}$,

$$
\left|u_{\lambda}(t, x, y)\right| \leq c_{14, q} \lambda^{q \max \left(\mu-\frac{2}{k}, 2 \alpha-\frac{2}{k}\right)-q \alpha+\alpha+\frac{1}{2}}\left|x^{2}+t^{2}\right|^{-\frac{q}{2}} .
$$

We deduce by getting back to (3.4.17), using (3.4.22) and a change of variables that for all $q \in \mathbb{N} \backslash\{0,1\}$ and $\lambda \geq \lambda_{0}$,

$$
\begin{aligned}
& \left\|\left(1-\chi_{1}\left(\lambda^{\gamma} t, \lambda^{\gamma} x\right)\right) u_{\lambda}\right\|_{\left(-N_{0}\right)} \\
\leq & c_{14, q}\left(c_{1}-c_{2}\right)^{\frac{1}{2}} \lambda^{q \max \left(\mu-\alpha-\frac{2}{k}, \alpha-\frac{2}{k}\right)+\alpha+\frac{1}{2}-\frac{1}{k}}\left\|1-\chi_{1}\right\|_{L^{\infty}\left(\mathbb{R}^{2}\right)} \\
& \times\left(\int_{\left\{(t, x) \in \mathbb{R}^{2}: t^{2}+x^{2} \geq 4^{-1} \lambda^{-2 \gamma}\right\}}\left|t^{2}+x^{2}\right|^{-q} d t d x\right)^{\frac{1}{2}} \\
\leq & c_{14, q}\left(c_{1}-c_{2}\right)^{\frac{1}{2}} \lambda^{q \max \left(\mu-\alpha-\frac{2}{k}+\gamma, \alpha+\gamma-\frac{2}{k}\right)+\alpha+\frac{1}{2}-\frac{1}{k}-\gamma}\left\|1-\chi_{1}\right\|_{L^{\infty}\left(\mathbb{R}^{2}\right)} \\
& \times\left(\int_{\left\{(t, x) \in \mathbb{R}^{2}: t^{2}+x^{2} \geq 4^{-1}\right\}}\left|t^{2}+x^{2}\right|^{-q} d t d x\right)^{\frac{1}{2}} .
\end{aligned}
$$

We recall in view of (3.1.5) that $c_{1}>c_{2}$. Let us notice that

$$
\max \left(\mu-\alpha-\frac{2}{k}+\gamma, \alpha+\gamma-\frac{2}{k}\right)<0,
$$

because from (3.4.13),

$$
\alpha+\gamma<\frac{2}{k}
$$

and from (3.1.3) and (3.4.13),

$$
\mu-\alpha-\frac{2}{k}+\gamma<\gamma-\frac{1}{k}<0 .
$$


Finally, we obtain using (3.4.23) and (3.4.24) the estimate (3.4.15). This ends the proof of the lemma 3.4.3.

We can now use (3.4.12) and the triangular inequality for all $\lambda \geq \lambda_{0}$,

$$
\left\|u_{\lambda}\right\|_{\left(-N_{0}\right)}-\left\|\left(1-\chi_{1}\left(\lambda^{\gamma} t, \lambda^{\gamma} x\right)\right) u_{\lambda}\right\|_{\left(-N_{0}\right)} \leq\left\|v_{\lambda}\right\|_{\left(-N_{0}\right)},
$$

with the estimates (3.3.14) and (3.4.15) to prove the following result.

Proposition 3.4.1 There exists a positive constant $c_{15}$ such that for all $\lambda \geq \lambda_{0}$,

$$
\left\|v_{\lambda}\right\|_{\left(-N_{0}\right)} \geq c_{15} \lambda^{-\alpha-1+\frac{\mu}{2}-(\mu+3+3 \alpha) N_{0}} .
$$

We now need to get an upper bound for the quantity $\left\|L_{2}^{*} v_{\lambda}\right\|_{\left(N_{0}\right)}, N_{0} \in \mathbb{N}$, with respect to the parameter $\lambda$. It follows from (3.1.6) and (3.4.12) that for all $\lambda \geq \lambda_{0}$,

$$
\begin{aligned}
L_{2}^{*} v_{\lambda} & =\chi_{1}\left(\lambda^{\gamma} t, \lambda^{\gamma} x\right) L_{2}^{*} u_{\lambda}-\lambda^{2 \gamma} \theta_{k}(y)\left(\partial_{x}^{2} \chi_{1}\right)\left(\lambda^{\gamma} t, \lambda^{\gamma} x\right) u_{\lambda} \\
& -2 \lambda^{\gamma} \theta_{k}(y)\left(\partial_{x} \chi_{1}\right)\left(\lambda^{\gamma} t, \lambda^{\gamma} x\right) \partial_{x} u_{\lambda}+\lambda^{2 \gamma}\left(\partial_{t}^{2} \chi_{1}\right)\left(\lambda^{\gamma} t, \lambda^{\gamma} x\right) u_{\lambda} \\
& +2 \lambda^{\gamma}\left(\partial_{t} \chi_{1}\right)\left(\lambda^{\gamma} t, \lambda^{\gamma} x\right) \partial_{t} u_{\lambda} .
\end{aligned}
$$

We note respectively $A_{\lambda}, B_{\lambda}, C_{\lambda}, D_{\lambda}$ and $E_{\lambda}$ the terms appearing in the right-hand-side of the last expression. Let us first notice that these five terms are $C^{\infty}$ on $\mathbb{R}^{3}$. Indeed, we have already proved after (3.1.15) that $u_{\lambda} \in C^{\infty}\left(\mathbb{R}_{y}, \mathcal{S}\left(\mathbb{R}_{t, x}^{2}\right)\right)$ and, it follows from (3.1.1), (3.1.5) and (3.4.16) that for all $(t, x) \in \mathbb{R}^{2}, \lambda \geq \lambda_{0}$,

$$
\operatorname{supp} u_{\lambda}(t, x, \cdot) \subset\left[-c_{1} \lambda^{-\frac{2}{k}},-c_{2} \lambda^{-\frac{2}{k}}\right]
$$

and from (3.1.6), $\theta_{k}(y)=(-y)^{k}$ if $y \in \mathbb{R}_{-}$. Moreover, we have already proved in (3.1.16) that $L_{2}^{*} u_{\lambda}$ is $C^{\infty}$ on $\mathbb{R}^{3}$. Then, we want to get an upper bound for the $H^{N_{0}}\left(\mathbb{R}^{3}\right)$ norm of the term $A_{\lambda}$. To do this, it is enough to get an upper bound for the quantity

$$
\left\|\partial_{t, x}^{M_{1}}\left(\chi_{1}\left(\lambda^{\gamma} t, \lambda^{\gamma} x\right)\right) \partial_{t, x, y}^{M_{2}}\left(L_{2}^{*} u_{\lambda}\right)\right\|_{L^{2}\left(\mathbb{R}^{3}\right)},
$$

where $M_{1} \in \mathbb{N}^{2}$ and $M_{2} \in \mathbb{N}^{3}$ verify $\left|M_{1}\right|+\left|M_{2}\right|=N_{0}$. Since

$\left\|\partial_{t, x}^{M_{1}}\left(\chi_{1}\left(\lambda^{\gamma} t, \lambda^{\gamma} x\right)\right) \partial_{t, x, y}^{M_{2}}\left(L_{2}^{*} u_{\lambda}\right)\right\|_{L^{2}\left(\mathbb{R}^{3}\right)} \leq \lambda^{\gamma\left|M_{1}\right|}\left\|\partial_{t, x}^{M_{1}} \chi_{1}\right\|_{L^{\infty}\left(\mathbb{R}^{2}\right)}\left\|L_{2}^{*} u_{\lambda}\right\|_{\left(\left|M_{2}\right|\right)}$,

it follows that there exists a positive constant $c_{16}$ such that for all $\lambda \geq \lambda_{0}$,

$$
\left\|A_{\lambda}\right\|_{\left(N_{0}\right)} \leq c_{16} \lambda^{\gamma N_{0}}\left(\sup _{|j| \leq N_{0}}\left\|\partial_{t, x}^{j} \chi_{1}\right\|_{L^{\infty}\left(\mathbb{R}^{2}\right)}\right)\left\|L_{2}^{*} u_{\lambda}\right\|_{\left(N_{0}\right)} .
$$


Thus, we deduce from (3.1.3), (3.2.18) and (3.4.28) that

$$
\left\|A_{\lambda}\right\|_{\left(N_{0}\right)}=O\left(e^{-\frac{c_{3}}{16} \lambda^{2\left(\alpha+\frac{1}{k}-\mu\right)}}\right) \text { when } \lambda \rightarrow+\infty .
$$

Let us now consider $\left(j_{1}, j_{2}, j_{3}\right) \in \mathbb{N}^{3}$. It follows from (3.4.16) that for all $\lambda \geq \lambda_{0}$

$$
\begin{aligned}
& \partial_{t}^{j_{1}} \partial_{x}^{j_{2}} \partial_{y}^{j_{3}} u_{\lambda}(t, x, y)=\frac{i^{j_{1}+j_{2}}}{(2 \pi)^{2}} \lambda^{\alpha+\frac{1}{2}+j_{1} \alpha+j_{2}(1+\alpha)} \int_{\mathbb{R}^{2}} e^{i\left(\lambda^{1+\alpha} x \xi+\lambda^{\alpha} t \tau\right)} \\
& \quad \times \tau^{j_{1}} \xi^{j_{2}} \psi_{1}(\tau, \xi) \partial_{y}^{j_{3}}\left[\chi_{0}\left(\lambda^{\mu}\left(y+\left(\lambda^{-1} \xi^{-1} \tau\right) \frac{2}{k}\right)\right) e^{-\Phi_{\lambda}(\tau, \xi, y)}\right] d \tau d \xi
\end{aligned}
$$

We can make again some integrations by parts in (3.4.30) as in (3.4.18). Thus, we obtain that for all $q \in \mathbb{N},(t, x) \neq(0,0), y \in \mathbb{R}$ and $\lambda \geq \lambda_{0}$,

$$
\begin{aligned}
& \partial_{t}^{j_{1}} \partial_{x}^{j_{2}} \partial_{y}^{j_{3}} u_{\lambda}(t, x, y) \\
= & \frac{i^{j_{1}+j_{2}}}{(2 \pi)^{2}} \lambda^{\alpha+\frac{1}{2}+j_{1} \alpha+j_{2}(1+\alpha)}\left(i \lambda^{1+\alpha} x+\lambda^{\alpha} t\right)^{-q} \int_{\mathbb{R}^{2}} e^{i\left(\lambda^{1+\alpha} x \xi+\lambda^{\alpha} t \tau\right)}\left(i \partial_{\tau}-\partial_{\xi}\right)^{q} \\
& \left(\tau^{j_{1}} \xi^{j_{2}} \psi_{1}(\tau, \xi) \partial_{y}^{j_{3}}\left[\chi_{0}\left(\lambda^{\mu}\left(y+\left(\lambda^{-1} \xi^{-1} \tau\right)^{\frac{2}{k}}\right)\right) e^{-\Phi_{\lambda}(\tau, \xi, y)}\right]\right) d \tau d \xi .
\end{aligned}
$$

Let us prove the following lemma.

Lemma 3.4.5 For all $\rho \in \mathbb{N}^{2}, j_{3} \in \mathbb{N}$, there exists a positive constant $c_{17, \rho, j_{3}}$ such that for all $(\tau, \xi) \in[1,4]^{2}, \lambda \geq \lambda_{0}$ and $y \in \operatorname{supp} \chi_{0}\left(\lambda^{\mu}\left(\cdot+\left(\lambda^{-1} \xi^{-1} \tau\right)^{\frac{2}{k}}\right)\right)$,

$$
\begin{array}{r}
\left|\partial_{\tau, \xi}^{\rho} \partial_{y}^{j_{3}}\left[\chi_{0}\left(\lambda^{\mu}\left(y+\left(\lambda^{-1} \xi^{-1} \tau\right)^{\frac{2}{k}}\right)\right) e^{-\Phi_{\lambda}(\tau, \xi, y)}\right]\right| \\
\leq c_{17, \rho, j_{3}} \lambda^{(2+2 \alpha+\mu) j_{3}+|\rho| \max \left(2 \alpha-\frac{2}{k}, \mu-\frac{2}{k}\right)} .
\end{array}
$$

Proof. The Leibniz formula first proves that

$$
\begin{aligned}
\partial_{y}^{j_{3}}\left[\chi _ { 0 } \left(\lambda^{\mu}(y+\right.\right. & \left.\left.\left.\left(\lambda^{-1} \xi^{-1} \tau\right)^{\frac{2}{k}}\right)\right) e^{-\Phi_{\lambda}}\right]= \\
& \sum_{l=0}^{j_{3}} C_{j_{3}}^{l} \lambda^{l \mu} \chi_{0}^{(l)}\left(\lambda^{\mu}\left(y+\left(\lambda^{-1} \xi^{-1} \tau\right)^{\frac{2}{k}}\right)\right) \partial_{y}^{j_{3}-l}\left(e^{-\Phi_{\lambda}}\right) .
\end{aligned}
$$

We deduce from the lemma 3.2.1 and (3.4.33) that there exist some functions $a_{r, l}, r=0, \ldots, j_{3}(k+1), l=0, \ldots, j_{3}$, which are polynomial in $\mathbb{R}^{4}$ and some constants $\beta_{r, l}, r=0, \ldots, j_{3}(k+1), l=0, \ldots, j_{3}$, verifying

$$
\beta_{r, l} \leq 2 j_{3}(\alpha+1)
$$


such that for all $y \in \mathbb{R}_{-}^{*},(\tau, \xi) \in \mathbb{R}_{+}^{*} \times \mathbb{R}_{+}^{*}$ and $\lambda \geq 1$,

$$
\begin{aligned}
& \partial_{y}^{j_{3}}\left[\chi_{0}\left(\lambda^{\mu}\left(y+\left(\lambda^{-1} \xi^{-1} \tau\right)^{\frac{2}{k}}\right)\right) e^{-\Phi_{\lambda}}\right]= \\
& \sum_{\substack{0 \leq l \leq j_{3} \\
0 \leq r \leq j_{3}(k+1)}} \chi_{0}^{(l)}\left(\lambda^{\mu}\left(y+\left(\lambda^{-1} \xi^{-1} \tau\right)^{\frac{2}{k}}\right)\right) y^{r} a_{r, l}\left(\tau^{\frac{1}{k}}, \tau^{-\frac{1}{k}}, \xi^{\frac{1}{k}}, \xi^{-\frac{1}{k}}\right) \lambda^{l \mu+\beta_{r, l}} e^{-\Phi_{\lambda}} .
\end{aligned}
$$

Since using the Leibniz formula on (3.4.35), we can write

$$
\begin{aligned}
\partial_{\tau, \xi}^{\rho} \partial_{y}^{j_{3}}\left[\chi _ { 0 } \left(\lambda^{\mu}(y+\right.\right. & \left.\left.\left.\left(\lambda^{-1} \xi^{-1} \tau\right)^{\frac{2}{k}}\right)\right) e^{-\Phi_{\lambda}}\right]= \\
\sum_{l, r, \rho_{1}, \rho_{2}, \rho_{3}} & {\left[c_{18, l, r, \rho_{1}, \rho_{2}, \rho_{3}} \partial_{\tau, \xi}^{\rho_{1}}\left(\chi_{0}^{(l)}\left(\lambda^{\mu}\left(y+\left(\lambda^{-1} \xi^{-1} \tau\right)^{\frac{2}{k}}\right)\right)\right)\right.} \\
& \left.\times y^{r} \partial_{\tau, \xi}^{\rho_{2}}\left(a_{r, l}\left(\tau^{\frac{1}{k}}, \tau^{-\frac{1}{k}}, \xi^{\frac{1}{k}}, \xi^{-\frac{1}{k}}\right)\right) \partial_{\tau, \xi}^{\rho_{3}}\left(e^{-\Phi_{\lambda}}\right) \lambda^{l \mu+\beta_{r, l}}\right],
\end{aligned}
$$

where the above sum is taken on $0 \leq l \leq j_{3}, 0 \leq r \leq j_{3}(k+1),\left(\rho_{1}, \rho_{2}, \rho_{3}\right) \in$ $\left(\mathbb{N}^{2}\right)^{3}, \rho_{1}+\rho_{2}+\rho_{3}=\rho$ and where $c_{18, l, r, \rho_{1}, \rho_{2}, \rho_{3}}$ are some constants, we deduce from (3.1.5), (3.4.8), (3.4.19) and (3.4.34), that there exist some positive constant $c_{19, l, r, \rho_{1}, \rho_{2}, \rho_{3}}$ and $c_{20}$ such that for all $(\tau, \xi) \in[1,4]^{2}, \lambda \geq \lambda_{0}$ and $y \in \operatorname{supp} \chi_{0}\left(\lambda^{\mu}\left(\cdot+\left(\lambda^{-1} \xi^{-1} \tau\right)^{\frac{2}{k}}\right)\right)$,

$$
\begin{aligned}
& \left|\partial_{\tau, \xi}^{\rho} \partial_{y}^{j_{3}}\left[\chi_{0}\left(\lambda^{\mu}\left(y+\left(\lambda^{-1} \xi^{-1} \tau\right)^{\frac{2}{k}}\right)\right) e^{-\Phi_{\lambda}}\right]\right| \\
\leq & \sum_{l, r, \rho_{1}, \rho_{2}, \rho_{3}} c_{19, l, r, \rho_{1}, \rho_{2}, \rho_{3}} \lambda^{\left|\rho_{1}\right|\left(\mu-\frac{2}{k}\right)} \lambda^{2\left|\rho_{3}\right|\left(\alpha-\frac{1}{k}\right)} \lambda^{l \mu+2 j_{3}(\alpha+1)} \\
\leq & c_{20} \lambda^{j_{3}(2+2 \alpha+\mu)+|\rho| \max \left(2 \alpha-\frac{2}{k}, \mu-\frac{2}{k}\right)},
\end{aligned}
$$

where the sum of the previous expression is taken on $0 \leq l \leq j_{3}, 0 \leq r \leq$ $j_{3}(k+1),\left(\rho_{1}, \rho_{2}, \rho_{3}\right) \in\left(\mathbb{N}^{2}\right)^{3}, \rho_{1}+\rho_{2}+\rho_{3}=\rho$. This proves (3.4.32) and ends the proof of the lemma 3.4.5.

Thus, since from (3.1.1) and (3.1.3),

$$
\text { supp } \psi_{1} \subset[1,4]^{2} \text { and } \max \left(2 \alpha-\frac{2}{k}, \mu-\frac{2}{k}\right)>0 \text {, }
$$

we can deduce from (3.4.31) and the previous lemma that for all $q \in \mathbb{N}$, there exists a positive constant $c_{21, q}$ such that for all $(t, x) \neq(0,0), y \in \mathbb{R}$ and $\lambda \geq \lambda_{0}$,

$$
\begin{aligned}
& \left|\partial_{t}^{j_{1}} \partial_{x}^{j_{2}} \partial_{y}^{j_{3}} u_{\lambda}(t, x, y)\right| \leq \\
& c_{21, q} \lambda^{\alpha+\frac{1}{2}+j_{1} \alpha+j_{2}(1+\alpha)+j_{3}(\mu+2+2 \alpha)+q \max \left(2 \alpha-\frac{2}{k}, \mu-\frac{2}{k}\right)-q \alpha}\left|t^{2}+x^{2}\right|^{-\frac{q}{2}} .
\end{aligned}
$$


It follows from (3.4.27) and from (3.1.6), $\theta_{k}(y)=(-y)^{k}$ for all $y \in \mathbb{R}_{-}$that there exists a positive constant $c_{22}$ such that we have the following estimate of the $H^{N_{0}}\left(\mathbb{R}^{3}\right)$ norm of the terms $B_{\lambda}, C_{\lambda}, D_{\lambda}$ and $E_{\lambda}$ defined in (3.4.26),

$$
\begin{aligned}
& \max \left(\left\|B_{\lambda}\right\|_{\left(N_{0}\right)},\left\|C_{\lambda}\right\|_{\left(N_{0}\right)},\left\|D_{\lambda}\right\|_{\left(N_{0}\right)},\left\|E_{\lambda}\right\|_{\left(N_{0}\right)}\right) \\
\leq & c_{22} \sum_{\beta_{1}, \beta_{2}}\left\|\partial_{t, x}^{\beta_{1}}\left(\chi_{1}\left(\lambda^{\gamma} t, \lambda^{\gamma} x\right)\right) \partial_{t, x, y}^{\beta_{2}} u_{\lambda}\right\|_{L^{2}\left(\mathbb{R}^{3}\right)^{3}}
\end{aligned}
$$

where the sum is taken on $\beta_{1}=\left(l_{1}, l_{2}\right), \beta_{2}=\left(l_{3}, l_{4}, l_{5}\right)$ with $l_{j}, j=1, \ldots, 5$ some integers verifying $0 \leq l_{j} \leq N_{0}+2$ and $l_{1}+l_{2} \geq 1$. Using these notations, let us consider some integers $l_{j}, j=1, \ldots, 5$ verifying

$$
0 \leq l_{j} \leq N_{0}+2 \text { and } l_{1}+l_{2} \geq 1 \text {. }
$$

We set $\beta_{1}=\left(l_{1}, l_{2}\right)$ and $\beta_{2}=\left(l_{3}, l_{4}, l_{5}\right)$. Since from (3.4.14) and (3.4.38),

$$
\operatorname{supp} \partial_{t, x}^{\beta_{1}} \chi_{1} \subset\left\{(t, x) \in \mathbb{R}^{2}: t^{2}+x^{2} \geq 1 / 4\right\},
$$

we deduce from (3.4.27) and (3.4.36) that for all $q \geq 2$, there exists a positive constant $c_{23, q}$ such that for all $\lambda \geq \lambda_{0}$,

$$
\begin{aligned}
& \left\|\partial_{t, x}^{\beta_{1}}\left(\chi_{1}\left(\lambda^{\gamma} t, \lambda^{\gamma} x\right)\right) \partial_{t, x, y}^{\beta_{2}} u_{\lambda}\right\|_{L^{2}\left(\mathbb{R}^{3}\right)} \\
\leq & c_{23, q} \lambda^{\alpha+\frac{1}{2}+\gamma\left(l_{1}+l_{2}\right)+\alpha l_{3}+(1+\alpha) l_{4}+(\mu+2+2 \alpha) l_{5}+q \max \left(\alpha-\frac{2}{k}, \mu-\alpha-\frac{2}{k}\right)}\left\|\partial_{t, x}^{\beta_{1}} \chi_{1}\right\|_{L^{\infty}\left(\mathbb{R}^{2}\right)} \\
& \times\left(\int_{-c_{1} \lambda^{-\frac{2}{k}}}^{-c_{2} \lambda^{-\frac{2}{k}}}\left(\int_{\left\{(t, x) \in \mathbb{R}^{2}: t^{2}+x^{2} \geq 4^{-1} \lambda^{-2 \gamma}\right\}}\left|t^{2}+x^{2}\right|^{-q} d t d x\right) d y\right)^{\frac{1}{2}} .
\end{aligned}
$$

Since with our choice of the integers $l_{j}$ in (3.4.38), we have

$$
\gamma\left(l_{1}+l_{2}\right)+\alpha l_{3}+(1+\alpha) l_{4}+(\mu+2+2 \alpha) l_{5} \leq\left(N_{0}+2\right)(3+2 \gamma+4 \alpha+\mu) .
$$

We deduce from (3.4.38), (3.4.39), (3.4.40) and a change of variables that

$$
\begin{aligned}
& \left\|\partial_{t, x}^{\beta_{1}}\left(\chi_{1}\left(\lambda^{\gamma} t, \lambda^{\gamma} x\right)\right) \partial_{t, x, y}^{\beta_{2}} u_{\lambda}\right\|_{L^{2}\left(\mathbb{R}^{3}\right)} \\
\leq & c_{23, q}\left(c_{1}-c_{2}\right)^{\frac{1}{2}} \lambda^{\alpha+\frac{1}{2}-\frac{1}{k}-\gamma+\left(N_{0}+2\right)(3+2 \gamma+4 \alpha+\mu)+q \max \left(\alpha-\frac{2}{k}, \mu-\alpha-\frac{2}{k}\right)+q \gamma} \\
& \left(\sup _{\left|\beta_{1}\right| \leq 2 N_{0}+4}\left\|\partial_{t, x}^{\beta_{1}} \chi_{1}\right\|_{L^{\infty}\left(\mathbb{R}^{2}\right)}\right)\left(\int_{\left\{(T, X) \in \mathbb{R}^{2}: T^{2}+X^{2} \geq 4^{-1}\right\}}\left|T^{2}+X^{2}\right|^{-q} d T d X\right)^{\frac{1}{2}} .
\end{aligned}
$$

We recall that in view of (3.1.5), we have $c_{1}>c_{2}$. Then, it follows from (3.4.37) and (3.4.41) that for all $q \geq 2$, there exists a positive constant $c_{24, q}$ such that

$$
\begin{aligned}
\max & \left(\left\|B_{\lambda}\right\|_{\left(N_{0}\right)},\left\|C_{\lambda}\right\|_{\left(N_{0}\right)},\left\|D_{\lambda}\right\|_{\left(N_{0}\right)},\left\|E_{\lambda}\right\|_{\left(N_{0}\right)}\right) \\
& \leq c_{24, q} \lambda^{\alpha+\frac{1}{2}-\frac{1}{k}-\gamma+\left(N_{0}+2\right)(3+2 \gamma+4 \alpha+\mu)+q \max \left(\alpha+\gamma-\frac{2}{k}, \mu+\gamma-\alpha-\frac{2}{k}\right)} .
\end{aligned}
$$


Since from (3.1.3) and (3.4.13),

$$
\max \left(\alpha+\gamma-\frac{2}{k}, \mu+\gamma-\alpha-\frac{2}{k}\right)<0,
$$

because

$$
\mu-\alpha-\frac{1}{k}<0 \text { and } \gamma-\frac{1}{k}<0,
$$

we obtain from (3.4.26), (3.4.29), (3.4.42) and (3.4.43) that for all $M \in \mathbb{N}$, there exists a positive constant $C_{M}$ such that for all $\lambda \geq \lambda_{0}$,

$$
\left\|L_{2}^{*} v_{\lambda}\right\|_{\left(N_{0}\right)} \leq C_{M} \lambda^{-M}
$$

To sum up, we have built a family $\left(v_{\lambda}(t, x, y)\right)_{\lambda \geq \lambda_{0}}$ in (3.4.12), which is $C^{\infty}$ on $\mathbb{R}^{3}$ and has according to (3.4.14) and (3.4.27), its support in the compact set

$$
B\left(0, \lambda^{-\gamma}\right) \times\left[-c_{1} \lambda^{-\frac{2}{k}},-c_{2} \lambda^{-\frac{2}{k}}\right]
$$

The estimates obtained in (3.4.25) and (3.4.44), for all $\lambda \geq \lambda_{0}$,

$$
\begin{gathered}
\left\|v_{\lambda}\right\|_{\left(-N_{0}\right)} \geq c_{15} \lambda^{-\alpha-1+\frac{\mu}{2}-(\mu+3+3 \alpha) N_{0}}, \\
\forall M \in \mathbb{N}, \exists C_{M}>0,\left\|L_{2}^{*} v_{\lambda}\right\|_{\left(N_{0}\right)} \leq C_{M} \lambda^{-M},
\end{gathered}
$$

allow us to prove that no a priori estimates of the following type can hold

$$
\begin{aligned}
& \exists C_{0}>0, \exists N_{0} \in \mathbb{N}, \exists V_{0} \text { an open neighbourhood of } 0 \text { in } \mathbb{R}^{3} \text { such that } \\
& \qquad u \in C_{0}^{\infty}\left(V_{0}\right), C_{0}\left\|L_{2}^{*} u\right\|_{(k-3)} \geq\|u\|_{\left(-N_{0}\right)} .
\end{aligned}
$$

This proves that the operator $L_{2}$ is nonsolvable in any neighbourhood of 0 in $\mathbb{R}^{3}$ in the sense where there do not exist an integer $N_{0} \in \mathbb{N}$ and an open neighbourhood $V_{0}$ of 0 in $\mathbb{R}^{3}$ such that for all $f \in H^{N_{0}}\left(V_{0}\right)$, there exists $u \in H^{-k+3}\left(\mathbb{R}^{3}\right)$ such that

$$
L_{2} u=f \text { on } V_{0}
$$

\section{References}

[1] F. Colombini, L. Pernazza, F. Treves, Solvability and nonsolvability of second-order evolution equations, Hyperbolic problems and related topics, 111-120, Grad. Ser. Anal., Int. Press, Somerville, MA (2003).

[2] Yu.V. Egorov, On an example of a linear hyperbolic equation without solutions, C. R. Acad. Sci. Paris Sér. 1 Math. 317, no 12, 1149-1153 (1993). 
[3] Yu.V. Egorov, Sur un exemple d'équation linéaire hyperbolique n'ayant pas de solution, Journées "Equations aux Dérivées Partielles" (SaintJean-de-Monts, 1992), Exp. No. XIV, 6 pp., École Polytech., Palaiseau (1992).

[4] L. Hörmander, The analysis of Linear Partial Differential Operators, Springer-Verlag (1983-1985).

[5] N. Lerner, On a class of doubly characteristic operators with a principaltype behaviour, preprint (2006).

[6] G.A. Mendoza, G.A. Uhlmann, A sufficient condition for local solvability for a class of operators with double characteristics, Am. Jour. Math. 106, 187-217 (1984).

[7] G.A. Mendoza, G.A. Uhlmann, A necessary condition for local solvability for a class of operators with double characteristics, J. Funct. Anal. 52 , no. 2, 252-256 (1983).

[8] F. Treves, On a question of Louis Nirenberg, Math. Res. Lett. 10, no 5-6, 729-735 (2003).

Department of Mathematics, University of California, Evans Hall, Berkeley, CA 94720, USA

E-mail address: karel@math.berkeley.edu 